\title{
Soziale oder sozialistische Marktwirtschaft - Möglichkeiten zur Steuerung der wirtschaftlichen Entwicklung durch Verfassungsrecht und Verwaltungsrecht ${ }^{1}$
}

\author{
Von Christoph Müller
}

\section{Sozialismus und Marktwirtschaft}

Nach dem Zusammenbruch der UdSSR und der von ihr abhängigen Länder schien es vielen, als würde die Marktwirtschaft weltweit triumphieren. Allerdings gibt es nirgendwo eine reine Marktwirtschaft. Nahezu überall besteht ein "Dual-System" mit einer "gemischten Wirtschaft". Einem Sektor privater Wirtschaft steht immer ein öffentlicher Sektor gegenüber. Die "öffentliche Hand" reguliert durch ihre Infrastrukturpolitik Rahmenbedingungen des ökonomischen Systems. Auf vielfältige Weise interveniert sie in die marktwirtschaftlichen Abläufe. Sie nimmt auch direkt am Wirtschaftsleben teil. Auf der Ebene des Zentralstaats wie auch auf der Ebene der Gemeinden sind "Öffentliche Unternehmen" tätig (public utilities). In diesen Formen des Übergangs vom "Steuerstaat" zum "Unternehmerstaat" hat man früher gelegentlich eine Art von "Staatssozialismus" und von "Gemeindesozialismus" gesehen. Ob diese Bezeichnungen zutreffen, hängt von Faktoren $\mathrm{ab}$, die noch untersucht werden müssen.

Ohne Anspruch auf Vollständigkeit sollen einige Gründe für das Scheitern des Sozialismus-Modells der UdSSR zusammengetragen werden, um aus einer Analyse der Fehler mögliche Schlußfolgerungen für unsere eigenen, heutigen Aufgaben zu ziehen. Es gibt einige objektive Daten, die weltweit wirksam sind, und denen zuzuschreiben ist, daß sich das Leben der Individuen zwangsläufig immer mehr "vergesellschaftet". Dazu gehören die Phänome der Industrialisierung, der Urbanisierung, der Belastung der Umwelt mit Schadstoffen und der dramatische demographische Anstieg der Weltbevölkerung. Es ist offensichtlich, daß sich unter diesen Bedingungen die Probleme einer nachhaltig reproduktionsfähigen Weltgesellschaft nicht allein über den Markt lösen lassen. Wegen einer gravierenden Krise der öffentlichen Finanzen, aber auch wegen innerer Strukturschwächen der "öffentlichen Verwaltung" und der "öffentlichen Wirtschaft", wird gegenwärtig der "öffentliche Sektor" des Wirtschaftslebens weltweit zurückgedrängt. Aber auch die

1 Tagungsbeitrag für die International Conference on Development and Interaction of Economy, Science and Technology, and Law, Beijing, V.R. China, 10.-13. Mai 1994. Der Beitrag knüpft an das Regierungsprogramm der chinesischen "Öffnungspolitik" an, die offiziell als System einer "Sozialistischen Marktwirtschaft" definiert wird. Vgl. dazu den Beitrag zur Tagung dieser Konferenz von Ma Hong, Honorary Director des State Council Development Research Center. Die englische und die chinesische Version meines Beitrags sind noch nicht veröffentlicht. 
"neoliberale Theorie" will und kann das Phänomen der "Dualwirtschaft" nicht rückgängig machen. Dem "politischen System" kommt, auch und gerade auf dem Gebiet der Wirtschaft, eine regulierende Aufgabe zu. Es gibt ja nicht nur ein "Staatsversagen", sondern auch ein "Marktversagen". Die "vollkommene Konkurrenz", unter der ein Markt funktionieren könnte, ist bekanntlich eine reine Modellvorstellung. Wenn die menschliche Zivilisation eine Zukunft haben soll, wird es unumgänglich sein, neben dem Markt auch die "vergesellschafteten" Strukturen öffentlicher Infrastrukturpolitik und und "öffentlicher Wirtschaft" neu zum Gegenstand der Analyse zu machen. Dazu ist auch eine Fehleranalyse der "sozialistischen Idee" erforderlich. Der "Marxismus-Leninismus" hatte beansprucht, die Pläne einer sozialistischen Umgestaltung, schlecht und recht, aber immerhin "real", zum Existieren gebracht zu haben. Er ist nicht nur politisch, sondern vor allem auch ökonomisch gescheitert. Es genügt nicht, den "Stalinismus" oder den "Leninismus" anzuklagen. Es müssen auch Schwächen der Theorie von Marx und Engels untersucht werden.

\subsection{Unersetzlichkeit der Funktion des "Unternehmers" und der Strukturen formaler Organisation}

Marx und Engels standen noch im Bann der Französischen Revolution. Sie hatten die Neigung, sich die sozialistische Umwälzung, die sie kommen sahen, ähnlich vorzustellen, wie diese bürgerliche Revolution. Es gab aber fundamentale Unterschiede. Vor allem waren die "Unternehmer" der bürgerlichen Gesellschaft nicht in der Weise funktionslos geworden, wie der Feudaladel des Spätabsolutismus. Auch eine sozialistische Gesellschaft mußte die von der Bevölkerung des Landes benötigten Gebrauchsgüter produzieren. Sie mußte in Land- und Forstwirtschaft und im Bergbau die Urproduktion weiterführen, handwerkliche wie industrielle Produktionsprozesse organisieren und für eine Zirkulation und Distribution der Güter sorgen. Die Zahl der hierfür in Betracht kommenden Organisationsformen war eng beschränkt. Die Produktion in der sozialistischen Gesellschaft sollte "planmäßig" erfolgen und den vernünftigen Bedürfnissen der ganzen Bevölkerung dienen. Die Arbeiterklasse konnte aber nicht als Kollektiv handeln. Sie war auf herkömmliche Instrumente formaler Organisation angewiesen.

Engels hatte das gelegentlich eingeräumt: Wenn jemand "Organisation" sage, so setze er damit auch Autoritätsstrukturen in dieser Organisation voraus. Aber er vertagte die Lösung der Probleme der Zukunftsgesellschaft; erst müsse man die Macht erobern, alles andere werde sich später finden. Auch Marx hat sich immer geweigert, "mit der Zukunft fertig werden" zu wollen. Das klang vernünftig, weil er nicht über Dinge spekulieren wollte, die man noch nicht wissen konnte. Aber Marx und Engels hätten nicht spekulieren, sondern vielmehr nur einräumen müssen, daß auch eine sozialistische Gesellschaft nur "mit Wasser kochen" konnte. Sie wollten aber die Elemente der Kontinuität zwischen bürgerlicher und sozialistischer Gesellschaft nicht akzeptieren. Ich nehme an, daß hier die Philoscphie Hegels eine negative Rolle gespielt hat. Seine Vorstellung von einem "dialek- 
tischen Umschlag" schien es zu rechtfertigen, sich auf die "Kritik der bürgerlichen Gesellschaft" zu beschränken und die Lösung der praktischen Probleme dann einer "Negation der Negation" zu überlassen. Aber nichts rechtfertigte die Annahme, daß eine Aufhebung des Privateigentums an den Produktionsmitteln ein neues Prinzip hervorbrachte, wie die Produktion organisiert werden könne.

Als im Moment der Revolution in Rußland der mystische Umschlag ausblieb, mußte Lenin entscheiden, von Marx und Engels alleingelassen, wie er die praktischen Probleme lösen könnte. Dabei stand er unter dem Einfluß zweier miteinander ganz unvereinbarer, gegensätzlicher Vorstellungen: dem französischen "Kommune-Modell" und, wenn diese Kombination auch etwas eigenartig klingt, dem Vorbild der "Deutschen Reichspost".

Marx und Engels hatten nach der grausamen Niederschlagung des Aufstands in Paris den politisch verständlichen Wunsch, sich mit den Kommunarden zu solidarisieren. Es scheint, daß Marx durchaus einige Vorbehalte hatte, sich inhaltlich mit ihren Organisationsformen zu identifizieren. Aber seine Bemerkungen über die Kommune, die wohl nur als politische Unterstützung gemeint waren, begannen in der Phantasie der Arbeiterbewegung das Vakuum zu füllen, das die absichtlich noch offen gehaltenen Fragen hinterlassen hatten, wie die organisatorischen Strukturen der Zukunftsgesellschaft vielleicht aussehen könnten. So ist es zu einer voreiligen ideologischen Aufwertung des in keiner Hinsicht funktionsfähigen "Kommune-Modells" gekommen, das die russische Revolution von ihrer Anfangsphase an fehlgeleitet hat.

Praktisch verwendbarer, aber in anderer Hinsicht ebenfalls unzulänglich, war das andere Modell in Gestalt der Formen des "organisierten Kapitalismus". Weit davon entfernt, abzusterben, war der neuartige "Staatskapitalismus" dazu übergegangen, die Eisenbahnen und das Telegraphenwesen zu verstaatlichen und auf der Ebene des Staates und vor allem der Gemeinden, große "öffentliche Unternehmen" zu schaffen. Engels hatte gegenüber dem damals auch als "Staatssozialismus" bezeichneten System Bismarcks eine ambivalente Haltung. Es traf zu, wenn er feststellte, daß öffentliche Unternehmen wie Reichsbahn und Reichspost den Kapitalismus nicht aufhoben, sondern ihm nur gut brauchbare Infrastrukturen zur Verfügung stellten. Auf der anderen Seite konnte er nicht umhin, in diesen Vergesellschaftungsformen schon formale Organisationsstrukturen zu erkennen, die eine sozialistische Gesellschaft in einem anderen Kontext ohne tiefgreifende Änderungen für ihre Zwecke nutzen konnte. Der Begriff "Staatssozialismus" stammt von den bürgerlichen "Kathedersozialisten", wie Engels die noch im Wohlfahrtsstaat des Spätabsolutismus verankerte Nachhut der liberalen Bewegung ironisch bezeichnete. Die Arbeiterbewegung lehnte naturgemäß diesen bismarckischen "Staatssozialismus" genau so ab, wie sie das "soziale Königtum" Lorenz v. Steins abgelehnt hatte

Etwas anders sah die Situation bei den öffentlichen Unternehmen der Gemeinden aus. Auch hier waren es die bürgerlichen "Kathedersozialisten", namentlich der unermüdliche Bodenreformer Adolf Damaschke, der den "Gemeindesozialismus" propagierte, wenn er 
nicht sogar auch den Begriff geprägt hat. Die Gemeinden stellten planmäßig bestimmte "Einrichtungen" für die Bürger nach dem Prinzip der "Kostendeckung" zur Verfügung, betrieben keine "Profitmaximierung", sondern "Bedarfsdeckungswirtschaft" in den Formen der "einfachen" Reproduktion. So diente die Entwicklung des öffentlichen Personennahverkehrs einer Gemeinde nicht primär als Einnahmequelle für den Gemeindehaushalt, sondern war ein Instrument der Stadterschließung und wollte die Verkehrsströme im öffentlichen Interesse organisieren. Marx und Engels maßen der Gemeindeebene für die Umgestaltung der Gesamtgesellschaft keine strukturgebende Bedeutung zu. Aber der Begriff des "Gemeindesozialismus" bürgerte sich, im Gegensatz zum Begriff des "Staatssozialismus", in der Arbeiterbewegung rasch ein. ${ }^{2}$ Die Widersprüche zwischen dem überhaupt nicht funktionierenden Kommune-Modell und dem immerhin nicht untauglichen Modell großer öffentlicher, staatlicher oder kommunaler Unternehmen wurden in der UdSSR nicht ausgetragen, sondern nur verbal überbrückt, was zu immer kühneren Konstruktionen führte, die dazu dienten, die Realität zu verhüllen und ihr einen schöneren Schein zu verschaffen.

$\mathrm{Zu}$ einer die Revolution gefährdenden wirtschaftliche Katastrophe kam es, als Lenin das Privateigentum an den Produktionsmitteln aufhob. Zur Versorgung der Bevölkerung und zur Organisation der Produktionsprozesse war er auf die Zusammenarbeit mit den Landwirten, auf das technische Wissen der bürgerlichen Unternehmer und der Fachleute in den verschiedenen Wirtschaftszweigen angewiesen. Zwar kann eine "Vergesellschaftung" von Produktionsmitteln nötig sein, unter anderem dann, wenn wirtschaftliche Machtstrukturen die Marktgesetze aufheben. Nach einer früher ganz allgemeinen Ansicht, die heute von der neoliberalen Theorie in Frage gestellt wird, gehören Monopole, wenn sie nicht vermieden werden können, unter "soziale" Kontrolle. Auch das deutsche Grundgesetz sieht in Art. 15 die Möglichkeit vor, Grund und Boden, Naturschätze und Produktionsmittel zu vergesellschaften. Aber es war ein Fehlgriff, auch solche bürgerlichen Unternehmer, die eine konstruktive und produktive Arbeit leisteten, an der Erfüllung ihrer sinnvollen sozialen Funktion zu hindern.

In der Periode der "Neuen ökonomischen Politik" (NöP) wurden diese Fehler halbherzig eingestanden und zum Teil korrigiert. Es kam aber nicht zu einer Wende, die den Weg zu einer wirklichen "Dualwirtschaft", nun nicht mehr auf kapitalistischer, sondern auf einer sozialistischen Basis, eingeleitet hätte. Dabei hätte das politische System die Rahmenbedingungen des Marktsektors bestimmen und ein dauerhaftes Bündnis mit allen Schichten eingehen müssen, die bereit waren, am Aufbau einer sozialistischen Gesellschaft mitzuarbeiten. Lenins "Schritt zurück" war aber nur taktisch gemeint. Statt die "Neue ökonomi-

Vgl. dazu Christoph Müller, Kommunalpolitische Inf rastrukturen des Wohlfahtsstaats. Bemerkungen zum Thema "Munizipal-Sozialismus"; spanische Version: "La infraestructura cummunal del Estado de bienestar. Observaciones en torno al tema del "socialismo municipal", in Iztapalapa, Revista de Ciencias Sociales y Humanidades, México, 12 (1992), S. 11-52. Der deutsche Text von 1989 sowie die chinesische und russische Übersetzung sind bisher noch nicht veröffentlicht. 
sche Politik" dann wieder durch eine umfassende bürokratische Staatswirtschaft nach dem Modell der "Deutschen Reichspost" zu ersetzen, wäre es für die Zielsetzungen der sozialen Umgestaltung des Landes besser gewesen, die UdSSR hätte diesen privaten Marktsektor erhalten. Sie hätte sich darauf beschränken können, neue, geeignete Rahmenbedingungen zu schaffen, ihm unter den Perspektiven des vergesellschafteten Sektors Impulse zu vermitteln und die Richtung zu bestimmen, in der sich die Marktkräfte bewegen sollten. Von den möglichen Vergesellschaftungsformen wurde im wesentlichen nur die der "Verstaatlichung" genutzt, wenn man von den Experimenten mit dem Genossenschaftswesen absieht, das aber später immer mehr an Bedeutung verlor. Die Alternative einer "Kommunalisierung" kam wegen der Schwäche der Gemeindeselbstverwaltung im alten Rußland zunächst kaum in Betracht; sie wurde aber auch nicht angestrebt. Da ohne ausdifferenzierte Verwaltung die großen Aufgaben nicht bewältigt werden konnten, blieb Lenin nur der Ausweg, sich mit der verhaßten und verknöcherten "zaristischen" Verwaltung wieder zu arrangieren. Auch mit dem administrativen Kommando-Modell, das dabei entstand, ließen sich nur manche Aufgaben lösen. Das galt für die Erschließung des Landes mit Verkehrswegen, namentlich mit Eisenbahnen, ferner für das Revolutionsziel der "Elektrifizierung" und andere Großprojekte. In manchen Bereichen entstanden auf diesem zentralistischen Wege durchaus leistungsfähige Strukturen. Es gelang, eine effiziente Rüstungsindustrie und später eine innovative Weltraumindustrie aufzubauen. Aber die großen Leistungen in diesen Sektoren wurden nicht für die gesamte Volkswirtschaft nutzbar gemacht. Sobald die Gesellschaft komplexer geworden war, zeigte das Verwaltungssystem der UdSSR fatale Schwächen. Es war ein unbeweglicher Leviathan. Seine Funktionäre waren Nutznießer der bürokratischen Wucherungen geworden und konnten, an einer Reform dieses Systems nicht mehr interessiert, alle Veränderungen abblocken.

Die DDR hat sich diesem "Modell" angepaßt. Der Sozialismus ist hier nicht an den Stellen weitergebaut worden, wo er bereits unter den Bedingungen kapitalistischer Gesellschaften ansatzweise funktioniert hatte. Der bewährte "Gemeindesozialismus" wurde vielmehr ruiniert. Gestützt auf die Traditionen der "Städteordnung" des Frh. v. Stein aus dem Jahre 1808, mit der die Demokratisierung Deutschlands ihren Anfang genommen hatte, waren in der Weimarer Republik ausbaufähige Strukturen der Gemeindedemokratie entstanden. Die Kommunalverfassung hatte die wesentlichen Ziele vorgegeben. Die "Gemeindedemokratie" hatte dabei fast von selbst zu einem Wettstreit um die besten Formen des "Gemeindesozialismus" geführt, bei dem - was auf der nationalen und gesamtstaatlichen Ebene mißlang - bürgerliche Kräfte und Exponenten der Arbeiterbewegung gut zusammenarbeiteten. Ein typischer sozialdemokratischer "Munizipalsozialist" wie Max Brauer erwarb sich das Vertrauen auch der bürgerlichen Schichten Hamburgs. Etwas Ähnliches galt bis zum Ende der Weimarer Republik von dem "Verkehrsstadtrat" Ernst Reuter, der die Kommunalisierung des öffentlichen Nahverkehrs von Berlin systematisch weiterbetrieben hatte, für die vor dem Ersten Weltkrieg der "Kathedersozialist" und Schöpfer der Weimarer Reichsverfassung, Hugo Preuß, die Grundlage geschaffen hatte. In 
der DDR strebte das "Neue ökonomische System der Planung und Leitung", das in den 60er Jahren entwickelt wurde, eine Art sozialistischer Marktwirtschaft an und wollte auch den Gemeinden einen Teil ihrer früheren Finanzhohheit zurückgeben. Diese Pläne scheiterten aber an den damaligen politischen Rahmenbedingungen, vor allem der rettungslos zentralisierten Finanzplanung. Alle diese Ansätze wurden am Ende radikal zerstört und die Kommunen ganz dem etatistischen Zentralismus untergeordnet. Auch in der DDR hatte der "Gemeindesozialismus" keine Chance.

Das Sozialismus-Modell der UdSSR hat im Bereich der Verwaltung eine überzentralisierte und einseitige Kommandowirtschaft hervorgebracht. Sie stand in ihrer Leistungsfähigkeit tief unter dem Niveau des Managements in der privaten Wirtschaft. Die moderne Systemtheorie hat begründet, daß die Effizienz der Verwaltung im privaten Sektor wie im öffentlichen, in der Industriebürokratie wie in der Staatsbürokratie, davon abhängt, daß nicht die Zentrale alles an sich zieht und ihre Autorität dominiert, sondern daß das System an seiner "Peripherie" die Kompetenz ausbildet, die Aufgaben dezentral und selbständig zu erledigen. Unter dem leninschen Motto "Vertrauen ist gut, Kontrolle ist besser", entstand ein irrationales, lähmendes Kontrollsystem. Das Wunder des Umschlags war ausgeblieben: Der neue sozialistische "Inhalt" hatte nicht automatisch neue "Formen" der Verwaltung hervorgebracht. Die vorsichtigeren Wege des Experimentierens mit einer "sozialistischen Dualwirtschaft" wurden wegen ideologischer Sperren nicht rückhaltlos und zielstrebig verfolgt. So konnte die Systemschwäche nicht mehr rechtzeitig erkannt und korrigiert werden.

\subsection{Kontinuität zwischen bürgerlichem und sozialistischem Recht}

Was aber die eigenen, inneren Schwächen angeht, so konnte sich das administrative Kommandomodell in der UdSSR auch deshalb entwickeln, weil schon die Theoretiker des Marxismus die Funktion von Staat, Verwaltung und Rechtsordnung ganz einseitig bestimmt hatten. Eine moderne, dezentrale Verwaltung ist auf den festen Rahmen einer in sich konsistenten und die Teilsysteme umgreifenden Rechtsordnung angewiesen. Zwar hatten Marx und Engels gute Gründe, die Instrumentalisierung des Rechts zu kritisieren, wie sie für den Wirtschaftsliberalismus charakteristisch war. Die "Klassenjustiz" der bürgerlichen Gesellschaft (Ernst Fraenkel) verdiente ihr hartes Urteil. Aber auch hier wirkte sich wieder hemmend aus, daß Marx und Engels sich nahezu ausschließlich auf eine Kritik der "bürgerlichen Inhalte" des Rechts konzentriert hatten. Für die Probleme der "Formen" des Rechts, wie sie eine "allgemeine Rechtslehre" analysiert und dabei Elemente der Kontinuität zwischen den juridisch-organisatorischen Strukturen einer bürgerlichen und einer sozialistischen Gesellschaft einsichtig machen kann, brachten sie kaum Verständnis auf.

Zur Erklärung des Phänomens "Recht" gingen sie auf die Entstehung der "Warenform" im Güteraustausch zurück. Aber nur Teile der Rechtsordnung hatten etwas mit Kauf und 
Tausch zu tun. Es gibt zahlreiche Rechtsgebiete, die sich mit ganz anderen gesellschaftlichen Problemen befassen. So geht zum Beispiel das Verbot der Eheschließung unter engen Verwandten, das Rechtsgebot der "Exogamie", schon auf die Urgesellschaft zurück. Auch die heutigen judiziellen Grundrechte, die den einzelnen vor administrativer Willkür schützen sollen, haben ihre Wurzeln in vorbürgerlichen Verhältnissen und allgemeinen, tief menschlichen Bedürfnissen. Die Erklärung der Religion zur Privatsache, wie sie zuerst die Amerikanische Revolution ausgesprochen hatte (Georg Jellinek), steht zwar im Zusammenhang damit, da $\dot{\beta}$ eine neue Gesellschaft im Entstehen begriffen war. Sie brachte ein neuartiges "Individuum" hervor, das begann, sich aus den uralten totalitären Einbindungen in den kollektiven Gewissenszwang von "Stammes- und Staatsreligionen" zu befreien. Damit wurde ein erster Schritt getan, um das Ende der jahrtausendealten Zusammenhänge von Staat und Religion, von "Palast und Tempel", von "Thron" und "Altar" einzuleiten. Diese Entwicklungen, die noch längst nicht zum Abschluß gekommen sind, lassen sich aber ebenfalls nicht auf die Dimension der Produktion und Distribution von Gütern reduzieren.

Marx und Engels kannten das Römische Recht gut und wußten, daß die Warenform erheblich älter war als die "entwickelte" bürgerliche Gesellschaft, die mit dem modernen Wirtschaftsliberalismus entstanden war. Aber sie gaben sich der Illusion hin, als stünde der Warencharakter bei der Produktion und der Zirkulation von Gebrauchsgütern zur Disposition und könne bei einer sozialistischen Umgestaltung in irgendeiner Weise zurückgenommen werden. Sie gingen der Frage nicht nach, welche der überkommenen Rechtsformen brauchbare Bausteine einer Zukunftsgesellschaft sein könnten. Bei ihrer berechtigten Kritik am konkreten "Inhalt" des Rechts in der bürgerlichen Gesellschaft haben sie die Bedeutung der Kontinuität im Bereich des Rechts nicht richtig eingeschätzt. Es war kurzschlüssig, wie Eugenij Pashukanis aus dem von Marx erfaßten Zusammenhang einiger Rechtsformen mit der Warenform kurzerhand folgerte, mit der revolutionären Überwindung der bürgerlichen Gesellschaft in Rußland sei der Warencharakter der Produktion verschwunden, und damit sei auch das Recht zum Absterben verurteilt. Marx und Engels wären mit einer so umstandslosen Verwandlung ihrer konkreten Analysen in "zwerghafte Formeln" sicher nicht einverstanden gewesen. Wie bei den Fragen der Organisation der Produktion stellte sich auch hier heraus, was Hans Kelsen mit klarem Blick erkannt hatte, daß ein politisches System, das sich die radikale Umgestaltung der Gesellschaft zum Ziel gesetzt hatte, nicht weniger, sondern umgekehrt noch mehr auf die Formen des Rechts angewiesen war. Von einem Ende der Warenproduktion konnte keine Rede sein, weil eine sozialistische Gesellschaft die ökonomische Arbeitsteilung nicht wieder abschaffen konnte, die in dem ganzen Zeitraum seit der "neolithischen Revolution" bis heute sich allenthalben bewährt hatte. Die sowjetische Rechtstheorie schlug dann ins Gegenteil eines harten Positivismus um. Sie instrumentalisierte die neue Rechtsordnung nun ihrerseits nach den Bedürfnissen eines bürokatischen Zentralstaats, und das hat 
wesentlich zur Entfremdung der Bevölkerung von den sozialistischen Experimenten beigetragen.

Am heutigen Bestand unserer Rechtsformen haben Jahrtausende gearbeitet (Hans Kelsen). Da die Rechtsordnung nur eine äußerst beschränkte Zahl von organisatorischen Alternativen entwickeln kann, kommt auch eine sozialistische Rechtsordnung nicht umhin, von den überkommenen Rechtsformen Gebrauch zu machen. Die hoffnungsvollen Ansätze, in der DDR eine Art von "sozialistischer Marktwirtschaft" zu entwickeln, scheiterten im Grunde daran, daß das sowjetische Sozialismus-Modell die Elemente der Kontinuität zwischen bürgerlicher und sozialistischer Gesellschaft verkannt hatte. Neben anderen Folgen hatte das vor allem den Aufbau einer leistungsfähigen, dezentralen Verwaltung behindert. Dezentralisierung gilt zwar allgemein als erwünscht. Sie führt aber nur dann nicht zum Zusammenbruch des Gesamtsystems, wenn die relativ verselbständigten "Untersysteme" durch eine feste Rechtsordnung zusammengehalten werden und die lokalen Instanzen "im Rahmen" übergreif ender rechtlicher Strukturen operieren.

\section{2. "Soziale Marktwirtschaft" und "Neo-Liberalismus"}

Kann als funktionsfähig nur ein System gemischter Wirtschaft gelten, so unterscheiden sich die gegenwärtig existierenden. Wirtschaftssysteme aber beträchtlich unter dem Gesichtspunkt, wie groß jeweils der private und der öffentliche Sektor sind, vor allem aber danach, welche konkreten Ziele die öffentliche Hand mit ihrer Infrastrukturpolitik und ihren Öffentlichen Unternehmen verfolgt. Der öffentliche Sektor kann unbedeutend oder strukturbestimmend sein. Er kann das Ziel verfolgen, dem Privatkapital die Eroberung fremder Rohstoff- und Absatzmärkte zu erschließen; oder er kann versuchen, ein Gegenprinzip zur Profitwirtschaft zu bilden, um das Marktversagen zu kompensieren, soziale Spannungen auszugleichen und nach den Bedingungen einer nachhaltigen Reproduktion des eigenen Systems zu suchen. Wo im globalen Wirtschaftsaustausch das "NullSummen-Spiel" gilt, bei dem der Gewinn des einen im Verlust des anderen besteht, kann er versuchen, das eigene System auf die Gewinnerseite zu bringen; aber er kann sich auch darum bemühen, ein nachhaltig reproduktionsfähiges Modell der Weltwirtschaft zu organisieren, da dauerhaft auch die Wirtschaftssysteme der Einzelstaaten nur stabilisiert werden können, wenn die Ungleichgewichte der Weltwirtschaft abgebaut werden, wovon wir weit entfernt sind.

\subsection{Die Strukturen des "sozialen Rechtsstaats" in Deutschland}

Die "soziale Marktwirtschaft" der Bundesrepublik Deutschland zeigt Vorzüge, wenn man sie mit dem rücksichtslosen Neoliberalismus vergleicht, den die Chicago-Schule befürwortet. Zu den "sozialen" Zielsetzungen, die heute das politische System Deutschlands dem privaten Marktsektor vorgibt, gehören u.a. wirksame Regeln des Finanzausgleichs 
durch Bundesgesetz. Ein wirksames Steuersystem sorgt für eine beträchtliche finanzielle Umverteilung, und ein im ganzen recht tragfähiges soziales Netz hat bisher solide allgemeine Rahmenbedingungen der sozialen Sicherheit geschaffen. Eine aktive Politik der öffentlichen Infrastrukturen greift an vielen Stellen ein und schafft vom politischen System aus neue Rahmenbedingungen, die es dann wieder erlauben, Markttechniken zu verwenden. Auf vielfältige Weise interveniert die öffentliche Hand auch direkt in die Wirtschaft. Das Wirtschaftssystem Deutschlands kann als "organisierter" Kapitalismus bezeichnet werden (Andrew Shonfield). Die Verfassung verlangt von Bund, Ländern und Gemeinden, annähernd gleichartige Lebensverhältnisse in allen Teilen des Landes zu garantieren (Art. 20, 28, 72 GG). Das ist in der Vergangenheit im ganzen gut gelungen und kann wohl als der größte Erfolg des deutschen Sozialstaats betrachtet werden. Außerdem waren im Bereich der Rechtsordnung neben die Institute des Privatrechts zahlreiche öffentlich-rechtliche Formen der Regulation getreten. Das Recht des bürgerlich-rechtlichen Dienst- und Werkvertrags wurde zu einem modernen Arbeitsrecht ausgebaut und durch ein ausgedehntes Sozialverwaltungsrecht ergänzt. Neben das private Mietrecht trat unter den Nachkriegsbedingungen ein öffentliches System des Mieterschutzes und der Wohnraumbewirtschaftung. Parallel zum privaten Baurecht entwickelte sich ein umfassendes öffentliches Baurecht, das das Bauen, auch auf eigenem Grund und Boden, unter administrative Genehmigungsvorbehalte, Bedingungen und Auflagen stellte. Lokales und regionales Bauplanungsrecht schufen für die Bautätigkeit Rahmenbedingungen des öffentlichen Interesses. Weder in den USA noch in Europa noch in Japan zählt der Wohnungsbau zu den Ruhmesblättern der Marktwirtschaft (John K. Galbraith). Gestützt auf die europäische Bodenreformbewegung wurde in Deutschland die private Bautätigkeit durch öffentlichen, gemeinnützigen, kommunalen Wohnungsbau oder wenigstens durch öffentliches Kreditwesen oder durch steuerliche Begünstigung ergänzt, was hier vor und nach dem Ersten Weltkrieg zu bedeutenden Leistungen geführt hatte.

In diesen Entwicklungen eines modernen Verwaltungsrechts hatte Karl Renner, ein führender Vertreter des "Austromarxismus" und ehemaliger Präsident der Republik Österreich, Zeichen eines Übergangs von der privaten Marktwirtschaft zu stärker vergesellschafteten Formen gesehen und deutete diese öffentlichen-rechtlichen "Komplementärinstitute", die neben die Institutionen des Zivilrechts getreten waren, als Vorboten einer sozialistischen Gesellschaft. Das war eine unzulässige Vereinfachung, weil es auch Formen des Verwaltungsrechts gibt, die nicht den Bürger schützen, der von der zunehmenden faktischen Vergesellschaftung bedroht ist, sondern die ihn in den "organisierten Kapitalismus" nur besser einfügen. Aber wenn es zu einer Verschiebung der Grenzen des Dualsystem kam und Rahmenbedingungen einer sozialistischen Struktur sichtbar wurden, so konnten dieses neue Verwaltungsrecht und die inzwischen entstandenen öffentlichen Unternehmen eine wirksame Form des qualitativen Übergangs von einem liberalen zu einem sozialistischen Rechtsstaat darstellen. Unter den Schlägen der neoliberalen Kritik ist diese alte und bisher weithin akzeptierte Traditionslinie weitgehend abgerissen, dem 
Verwaltungsrecht und den öffentlichen Unternehmen der staatlichen und kommunalen Ebene die Aufgabe zu stellen, ein strukturelles Gegengewicht zur Profitwirtschaft zu bilden. Sozialdemokratie und Gewerkschaftsbewegung waren zu schwach, um gegenüber den allgemeinen Trends noch ein Gegenkonzept zu entwickeln und sich damit gegenüber der Bevölkerung verständlich zu machen.

\subsection{Vom Wirtschaftsliberalismus zum kapitalistischen Wohlfahrtsstaat}

Trotz mancher Vorteile der "sozialen Marktwirtschaft" der Bundesrepublik Deutschland gegenüber dem rücksichtslosen Neo-Liberalismus, läßt sich dieses Modell nicht ohne weiteres verallgemeinern. Das liegt nicht nur an seinen spezifischen historischen Rahmenbedingungen, sondern auch daran, daß es sich gegenwärtig selbst mit gravierenden Krisenerscheinungen auseinandersetzen muß. Es gibt einige spezifische Gründe, weshalb das Wirtschaftssystem Deutschlands, im Vergleich mit dem heute weltweit vorherrschenden Neo-Liberalismus, ursprünglich von einigen stark sozialen Zielsetzungen geprägt war. Das erklärt sich historisch einmal daraus, daß Deutschland die Entwicklung der liberalen Theorie, im Vergleich zu England, Frankreich und den USA, verspätet erlebt hat und Bismarck die liberale Bewegung im Heereskonflikt von 1862 gespalten und geschwächt hatte. Während Preußen noch ein Agrarstaat war, dachten zwar Kant und Hegel schon über die rückständigen Verhältnisse hinaus und verarbeiteten die neuen westeuropäischen Impulse. Aber die Deutschen waren, wie es Marx spöttisch formulierte, Zeitgenossen der wirklichen, sich modernisierenden Welt damals nur in der philosophischen Antizipation, noch nicht in der Realität. Auf der anderen Seite war der relativ aufgeklärte, aber ökonomisch noch wenig entwickelte, spätabsolutistische Wohlfahrtsstaat in Deutschland noch sehr stark. Die Theoretiker des "Kameralismus" und der "sozialen Monarchie" paßten den Liberalismus, als sie ihn dann zögernd aufnahmen, an die Vorstellungswelt des spätabsolutistischen Wohlfahrtsstaats an. So konnte - was zu den ironischen Wendungen der deutschen Geschichte gehört - die "Nachhut" der liberalen Bewegung zur "Avantgarde" des modernen Wohlfahrtsstaats werden.

Obwohl ein Teil des deutschen Bürgertums begann, sich mit der Entwicklung zum Verfassungsstaat auszusöhnen, hat es die demokratische Entwicklung Deutschlands im 19. Jahrhundert behindert, daß Arbeiterbewegung und Reformbürgertum nicht in der Lage waren, Sphären der Zusammenarbeit zu finden. Die Anfänge des deutschen "Sozialstaats" sind mit dem Namen Otto v. Bismarck verbunden, der einerseits die Sozialdemokraten mit einem Ausnahmegesetz unterdrückte und andererseits mit wohlfahrtsstaatlichen Maßnahmen versuchte, ihnen den Wind aus den Segeln zu nehmen. Der "proletarische Sozialismus" und der "bürgerliche Sozialismus" standen sich unversöhnlich gegenüber. Die marxistische Theorie lehnte eine Zusammenarbeit mit den bürgerlichen Reformbewegungen des "Kathedersozialismus" im allgemeinen ab. Diese ideologische Radikalisierung der sozialistischen Bewegung in Deutschland war ein Reflex der Tatsache, daß der preußisch- 
deutsche Obrigkeitsstaat keine absehbare Perspektive demokratischer Reformpolitik bot (Hermann Heller).

Das Schicksal der Weimarer Republik hing davon ab, ob es gelang, eine Zusammenarbeit von fortschrittlichem Bürgertum und Arbeiterbewegung zu finden (Hermann Heller). Das ist bekanntlich auf der gesamtstaatlichen Ebene gescheitert. Für ein Urteil über die Perspektiven der Weimarer Republik ist es aber nicht ohne Bedeutung, daß diese Zusammenarbeit auf der Ebene der Gemeinden auf vielfälige Weise gelungen ist. Waren auf der Ebene der staatlichen Wirtschaftspolitik alle Wege blockiert, die zu einer "sozialistischen Marktwirtschaft" hätten führen können, so kam es immerhin auf der kommunalen Ebene zu einer fruchtbaren Kooperation. Arbeiterbewegung und Reformbürgertum sahen in der Gemeindedemokratie gemeinsam eine Art von "Gemeindesozialismus", und beide bewerteten diese Tatsache positiv.

Statt daß sich die Arbeiterbewegung an die Spitze der reformbürgerlichen Bewegung setzte, ihre Halbheiten kritisierte, um in konkreten Projekten mit den potentiellen demokratischen Reformkräften zusammenzuarbeiten, fand - als Spätfolge der Bismarckschen Konfrontationspolitik - eine tragische Entfremdung zwischen beiden Schichten statt, was später das Aufkommen des Hitler-Regimes begünstigt hat. Diese alten Zuspitzungen haben auch nach 1945 die Entwicklungen zu einer "sozialistischen Marktwirtschaft" ungünstig beeinflußt, auch wenn die Unternehmer zu Zeiten durchaus auch Vorteile in einer starken sozialen Infrastrukturpolitik der öffentlichen Hand gesehen hatten, allerdings unter der Bedingung, daß "sozialistische Experimente" um jeden Preis vermieden wurden. In der Frühphase der CDU gab es einen nicht unbedeutenden Flügel, der sich im "Ahlener Programm" für umfassende Vergesellschaftung aussprach (Arkadius Gurland). Aber unter den Bedingungen der Nachkriegspolitik, des "Antikommunismus" und des Kalten Krieges war für solche Entwicklungen weder in Deutschland noch anderswo das Klima günstig. Die Sozialdemokratie, überrascht von ihrer Wahlniederlage, setzte auf das skandinavische Modell, gewann aber nur für kurze Zeit direkten Einfluß.

Nach dem Abenteuer des Hitlerfaschismus schloß sich Deutschland im großen und ganzen dem westeuropäischen und nordamerikanischen Modell des "Wohlfahrtsstaats" an, fügte aber mit dem werbewirksamen Begriff des "sozialen Rechtsstaats" und der "sozialen Marktwirtschaft" (Müller-Armack, Aleander Rüstow) eine besondere Note hinzu. Den Begriff des "sozialen Rechtsstaats" hatte in der Weimarer Republik der dem Austromarxismus nahestehende deutsche Staatstheoretiker Hermann Heller geprägt. Er wollte damit begründen, daß der liberale Rechtsstaat zum sozialistischen Rechtsstaat übergehen müsse, um einen Ausweg aus den Krisen zu finden, die dann bald zum deutschen Faschismus führen sollten ${ }^{3}$. Er war davon überzeugt, an diesem Punkte ähnlich wie Schumpeter, daß zwischen bürgerlicher und sozialistischer Gesellschaft durchaus ein unauflösli- 
cher Kulturzusammenhang bestand, insbesondere die rechtlichen und politischen Formen der bürgerlichen Gesellschaft für die Verwirklichung sozialistischer Ziele unabdingbar seien. Aber zugleich hielt er substantielle gesellschaftliche Umwälzungen für nötig, weil für ihn die "gesellschaftliche Form des Kapitalismus ein Vandalismus" war und es bei der "Anarchie der Produktion" unter kapitalistischer Reproduktionsbedingungen nötig blieb, "die Arbeitnehmer vor der Willkür und den Zufällen einer privatkapitalistischen Profitwirtschaft" zu schützen. Der neue Sprachgebrauch vom "sozialen Rechtsstaat", wie er sich nach 1949 in Deutschland durchsetzte, münzte den Begriff Hellers völlig um. Er wurde nun zum antikommunistischen Kampfbegriff und diente dazu, die staatlichen Interventionen in den Markt und die Leistungen der öffentlichen Hand für die Reproduktion des Gesamtsystems, die in großem Umfange stattfanden, hinter diesem Schlagwort und dem ins Englische kaum übersetzbaren "ordo"-Begriff (Ordo-Liberalismus) so zu verstecken, $\mathrm{da} ß$ auch sie im Bewußtsein der Bevölkerung kurzerhand dem "Markt" zugute gehalten wurden, obwohl diese politische Intervention an sich auf dem Gegenprinzip zur Selbstregulation des Marktes beruhte.

Der "Sozialstaat" der Bundesrepublik Deutschland ist in der Periode des "Kalten Krieges" nur von kleinen Gruppen als unzulänglich betrachtet worden. In der Meinung der Mehrheit hatte er sich bewährt.Auch Sozialdemokratie und Gewerkschaftsbewegung machten sich die "soziale" Marktwirtschaft ganz zu eigen. Dabei hat die "soziale" Marktwirtschaft in Deutschland die frühere "Gemeinwirtschaft" der Gewerkschaftsbewegung, den "gemeinnützigen" kommunalen Wohnungsbau und das Genossenschaftswesen weitgehend ruiniert. Die "Überflußgesellschaft" erschien der breiten Bevölkerung so attraktiv, daß Arbeiterbewegung und Gewerkschaften ihre alte Rolle, ein Gegenprinzip zur Profitmaximierung zu vertreten, praktisch preisgaben und sich darauf beschränkten, unter den Rahmenbedingungen, die sie vorfanden und nun auch hinnahmen, die Interessen der einfachen Bürger so gut wie möglich zu vertreten. Sie gaben ihre alten "sozialistischen" Zielsetzungen auf und sahen sich nicht imstande, unter den gewandelten Bedingungen das Ziel einer "sozialistischen Marktwirtschaft" zu entwickeln. Statt offensiv darauf zu bestehen, daß die öffentliche Sphäre weiterhin eine Gegenstruktur zur Profitwirtschaft bilden müsse und statt, was durchaus nötig war, die Funktionsweise der öffentlichen Unternehmen zu modernisieren, geben Sozialdemokraten und Gewerkschaftsbewegung nun auch dem neoliberalen Druck zur Privatisierung nach. In dieser Anpassung an den "Zeitgeist" liegt der tiefere Grund dafür, daß ihre eigenen "gemeinwirtschaftlichen Unternehmen" (Neue Heimat, Volksfürsoge, Coop) auch von innen durch Korruption zerstört werden konnten. Unter dem Einfluß des Antikommunismus, der sich am Ende gegen alles richtete, was direkt oder indirekt als "sozialistisch" bezeichnet werden konnte, haben Sozialdemokratie und Gewerkschaften die Kraft zum Gegensteuern weithin verloren.

Auch in Frankreich kam es nicht zur Entwicklung einer "sozialistischen Marktwirtschaft", obwohl hierfür einige Faktoren günstig waren. Starke Kräfte der alten merkantilistischen Wirtschaftspolitik und des absolutistischen Staatsverständnisses waren nach der Französi- 
schen Revolution eine enge Verbindung mit dem napoleonischen System eingegangen (Tocqueville). Das demokratische Reformbürgertum, namentlich in der sogenannten "Radikalsozialistischen" Partei, versuchte einen gewissen Brückenschlag zur Arbeiterbewegung. Das trug während der Dritten Republik zu einer starken Konsolidierung der Demokratie bei. Bis heute spielt in Frankreich der Staat als Wirtschaftsfaktor und Gegenspieler der Marktkräfte eine besondere Rolle. Leistungsfähige öffentliche Unternehmen haben hier noch eine relativ solide gesellschaftliche Basis. Aber trotz vieler positiver Experimente haben sich tragfähige Strukturen noch nicht gezeigt. Die Marktkräfte dominierten so stark, daß es nicht gelang, die Gegenkräfte zu stabilisieren. Weder der Volksfront-Regierung von León Blum 1936 noch der Koalition von Sozialisten mit Kommunisten der Regierung Mitterand war es möglich, den Grund für eine "sozialistische" Marktwirtschaft zu legen. In England war es relativ frühzeitig gelungen, unter der Einwirkung der "Fabian Society", die kulturelle Isolierung der Arbeiterbewegung zu überwinden. Was sie hervorgebracht hat, ist, auch wegen innerer Schwächen, dem Thatcherismus weitgehend zum Opfer gefallen. Auch in Skandinavien fand die Arbeiterbewegung breite gesellschaftliche Anerkennung. In diesem Klima konnten Reformkonzepte gedeihen und eine konstruktive Zusammenarbeit von Reformbürgertum und Arbeiterschaft möglich werden. Aber inzwischen stehen die sozialen Errungenschaften auch hier unter scharfer Kritik der neoliberalen Theorie. In den USA sind die interessanten Neuansätze des New Deal zur Zeit fast ganz abgerissen, obwohl sich jetzt wieder etwas "Glut unter der Asche" zeigt. Auch der "Austro-Marxismus", dessen Anziehungskraft darauf beruht hatte, daß Theoretiker wie Karl Renner und Otto Bauer über große praktische Erfahrung in Verwaltungsfragen verfügten, ist seit langem auf dem Rückzug. Die Rahmenbedingungen des Marktes, der Kapitalverzinsung und der Profitwirtschaft waren bei allen diesen Versuchen, den "rohen" Kapitalismus zu überwinden insgesamt so stark, daß sich bis heute in den westlichen Systemen das Konzept einer "sozialistischen Marktwirtschaft", die solide Gegenstrukturen zum Prinzip der Profitmaximierung hätte schaffen können, nicht hat entwickeln können. Wo es hierfür Ansatzpunkte gab, wurden sie in konzertierter Aktion totkonkurriert. Gegenwärtig befindet sich auch die "sozialere" Variante des Wirtschaftsliberalismus, die "soziale Marktwirtschaft" in der Defensive gegen die Angriffe der neoliberalen Chigaco-Schule. Erst die ökologische Krise beginnt, neue Akzente in die festgefahrene Diskussion zu bringen.

\subsection{Interne Krisenerscheinungen des westlichen Wohlfahrtsstaats}

Nach dem Zweiten Weltkrieg war in Skandinavien und in England die Theorie der Vollbeschäftigung entstanden (Beveridge, Full employment in a Free Society). Auch die NewDeal-Periode der USA hatte das gleiche Ziel verfolgt. Vollbeschäftigung wurde weithin als eine Alternative zum Sozialismus betrachtet. Etwa zwei Jahrzehnte konnte sie recht erfolgreich praktiziert werden. Heute aber ist sie zusammengebrochen. In der Bundesrepublik mit etwa 80 Millionen Einwohnern gibt es über vier Millionen Arbeitslose. Die 
Instrumente der "Konjunkturpolitik", wie sie vor allem John Maynhard Keynes systematisch entwickelt hatte, versagten. Danach sollte der Staat eine wirtschaftliche "Stagnation" dadurch bekämpfen, daß er die Konjunktur durch "fiskalische" Maßnahmen belebte, im wesentlichen durch direkte staatliche Ausgaben oder indirekt durch Maßnahmen zur Förderung von Investitionen. Eine Verschuldung der öffentlichen Hand und eine gewisse Geldentwertung wurden als unvermeidlich betrachtet und hingenommen. Inzwischen hat die Staatsverschuldung, auch und gerade der höchstentwickelten Industriestaaten, das $\mathrm{Ma} ß$ des Vorstellbaren überschritten. Vor allem versagten die Keynesianischen Instrumente, wenn Stagnation und Inflation gleichzeitig auftraten und wegen zu starker inflationärer Entwicklung ein großzügiges deficit spending der öffentlichen Hand und Erleichterungen für die Wirtschaft bei der Beschaffung von Krediten nicht mehr zu vertreten waren.

Die neo-liberale Theorie hat vor allem zwei Ratschläge, um die neuen ökonomischen Krisenerscheinungen der "Stag-Flation" zu beherrschen: Einmal verlangt sie, um die öffentliche Verschuldung zu bekämpfen, eine radikale Einschränkung der öffentlichen Ausgaben. Gleichzeitig drängte sie auf weitere Erhöhung der Arbeitsproduktivität, um die internationale Wettbewerbsfähigkeit zu stärken. Ökonomisch gesehen sind diese Ratschläge durchaus wirkungsvoll, jedenfalls solange man sie nur in ihren unmittelbaren betriebswirtschaftlichen Wirkungen betrachtet. Nicht umsonst hat Milton Friedman den Nobelpreis für Wirtschaft erhalten. Aber die sozialen Auswirkungen seiner Theorie sind intern für die einzelnen nationalen Wirtschaftssysteme ruinös; es entstanden die heutige Formen der Massenarbeitslosigkeit und eine ganz "neue" Armut. Vor allem hat sie katastrophale Auswirkungen auf die externen Zusammenhänge des internationalen Wirtschaftsaustauschs. Es hat sich nicht bewahrheitet, wie gehofft worden war, daß an Stelle der durch die Computer-Technologie freigesetzten Arbeitsplätze in ausreichendem Umfang neue entstehen würden. Die immer weitere Erhöhung der Arbeitsproduktivität macht Massenentlassungen möglich, die unter den Bedingungen der "internationalen Wettbewerbsfähigkeit" dann ökonomisch auch unvermeidlich werden. Die "sozialen Kosten" dieser Produktionsweise, die die Arbeiter überflüssig macht, können nicht mehr über den Markt aufgefangen werden. Wenn man sich einmal von allen Anwandlungen des Zeitgeistes freimacht, dann ist eigentlich nun weltweit die Situation entstanden, die Marx vor über einem Jahrhundert richtig antizipiert hatte und für die er nur noch den Ausweg einer "sozialistischen" Umverteilung sah. Während sozialistische Antworten immer dringlicher werden, sind ihre Rezepte aber hoffnungslos diskreditiert. Kein einzelnes Land findet einen Ausweg aus dem Dilemma. Das Marktversagen verlangt, diese Ungleichgewichte vom politischen System aus zu beheben. Aber angesichts der weltweiten Verflechtungen sind auch mächtige Nationalstaaten dazu nicht mehr in der Lage. Die am meisten industrialisierten Staaten entwickeln sich in ihrem Inneren zu "Zwei-Drittel-Gesellschaften". Gewaltige soziale Verwerf ungen geben Grund zu großer Beunruhigung. 
Es gibt eine Reihe von praktischen Vorschlägen, wie man den krisenhaften Entwicklungen abhelfen könnte. Sie reichen von der Forderung nach einer Besteuerung des Maschineneinsatzes über Versuche, die Kosten für den Einsatz von Arbeit zu senken, bis hin zum Vorschlag nach radikaler Arbeitszeitverkürzung. Aber es fehlt noch immer an einem umfassenden Konzept, das Kristallisationskern sein könnte, bei dem die vielfältigen neuen Ansatzpunkte eine kohärente Struktur annehmen könnten. Es fehlt ein Lösungsvorschlag, der im allgemeinen politischen Bewußtsein die Grundlage für den Aufbau einer alternativen "neue Gesellschaft" werden und positive, kreative Energien freisetzen könnte. Es herrscht eine allgemein Perspektivlosigkeit. Das heute nahezu totale Verdikt über alle Formen von sozialistischen Lösungen behindert dabei die Suche nach Auswegen.

\subsection{Externe Krisenerscheinungen der Weltwirtschaft}

Die heute vorherrschende neoliberale Theorie hat vor allem den gravierenden Nachteil, daß der internationale Wirtschaftsaustausch, wie sie ihn befürwortet, als Kampf der Wirtschaftsgiganten um "Wettbewerbsfähigkeit" und Marktanteile, nicht vernünftig funktioniert. Die Politik der Entwicklungshilfe, die nach dem Zweiten Weltkrieg eingeleitet worden ist, hat zwar einige positive Resultate erbracht, ihr eigentliches globales Ziel einer allseitigen Entwicklung aller Staaten der Erde aber nicht erreicht und kann es auf den bisherigen Wegen auch nicht erreichen. Weltweit hat die Verarmung zugenommen. Den pauperisierten Menschenmassen der Erde zeigen die neoliberalen Vorschläge keinen Weg. Im Gegenteil, der Konkurrenzdruck der ökonomisch herrschenden Weltkonzerne stranguliert die Entwicklung der Binnenwirtschaft vieler Länder. An dieser "internationalen Konkurrenz" sind viele Versuche gescheitert, durch eigene gewerbliche und industrielle Unternehmen ausländische Importe zu "substituieren".

Die marxistische Theorie hatte den Hauptwiderspruch der kapitalistischen Gesellschaft in dem unauflösbaren Widerspruch gesehen, der bei einer naturwüchsigen Reproduktion des Kapitals zu einer Verelendung der unmittelbaren Produzenten führen werde. Sie mache den Kapitalismus unhaltbar, an ihr werde er scheitern. Die westliche Wirtschaftswissenschaft war überzeugt, diese "Verelendungstheorie" widerlegt zu haben. Tatsächlich gab es schon zu Lebzeiten von Marx Anzeichen dafür, daß die Einkommen der englischen Industriearbeiter stiegen. Gleichwohl erscheint es zweifelhaft, ob Marx, der in Fragen der Staats-, Rechts- und Verwaltungstheorie manche Kritik verdient, sich an diesem Punkte wirklich geirrt hat. Denn er war nicht an einer einzelnen nationalen Volkswirtschaft interessiert, sondern antizipierte eine Weltwirtschaft, die alle Nationalstaaten in ihren Sog ziehen werde. In der Linie der Entwicklung der bürgerlichen Gesellschaft sah er voraus, was heute im Blick auf die internationalen Finanzströme nahezu der Fall ist, daß die "ganze Handelswelt eine Nation" ist. Marx und Engels haben sich den Wohlfahrtsstaat und den Konsum breiter Massen der Bevölkerung in den hochindustrialisierten Staaten nicht vorstellen können. Aber auch wenn wir einmal die Massenarbeitslosigkeit im Inneren der reichsten Staaten vernachlässigen, so hat jedenfalls im Weltmaßstab die Berüh- 
rung unentwickelter Länder mit der modernen Welt zu einer fast unvorstellbaren Verarmung geführt.

Der internationale Wirtschaftsaustausch hat in vielen Ländern die Bedingungen der traditionellen Lebensweise zerstört, ohne den Grund für eine moderne Gesellschaft zu legen. Ein kleiner Teil der Weltbevölkerung verbraucht den größten Teil der Ressourcen des Globus und produziert die Hauptmasse der Umweltbelastung, während die weit überwiegende Zahl der Bewohner des Planeten in Armut und Hoffnungslosigkeit versinkt. Dabei ist die physische und kulturelle Verelendung auch die Hauptursache des irrationalen Bevölkerungswachstums, das ganz überwiegend in der sogenannten "Dritten Welt" stattfindet. Man kann es niemandem verdenken, der ohne soziales Netz leben muß, daß er sich die Sicherheit seiner Existenz nur vorstellen kann, wenn viele Kinder im Kampf um das physische Überleben mitarbeiten und später die Versorgung der Alten übernehmen. Drei Milliarden Menschen leben heute ohne Elektrizität. Es gibt Schätzungen, daß im Jahre 2000 etwa zwei Milliarden Menschen auf dem Planeten auch das Brennholz nicht mehr finden werden, das sie zum Überleben brauchen, während sie zugleich durch Waldzerstörung ihre bisherigen Überlebensbedingungen untergraben.

Auch die höchstindustrialisierten Staaten befinden sich in einer Wirtschaftskrise. Es erscheint frivol, sie mit dem Elend der "Entwicklungsländer" in Vergleich zu setzen. Aber noch frivoler ist der Rat, den die neo-liberale Theorie erteilt, wie wir aus unserer "Rezessions-Krise" herauszukommen könnten: Das weitere inzwischen dysfunktional gewordene Wirtschaftswachstum soll in den gegebenen weltwirtschaftlichen Machtstrukturen weiter forciert werden. Sie macht keine brauchbaren Vorschläge, wie die weltwirtschaftlichen Ungleichgewichte überwunden werden könnten. Inzwischen ist es zu einer wahren "Völkerwanderung" gekommen. Die aktivsten Teile der pauperisierten Massen des Globus haben sich in Bewegung gesetzt. Sie üben einen Migrationsdruck auf die höchstindustrialisierten Staaten aus, während ihre eigene Gesellschaft ausgeblutet wird ("brain-drain"). Weit davon entfernt, sich auch an diesem Punkte den eigenen liberalen Prinzipien der "vollkommenen Konkurrenz" verpflichtet zu fühlen, zu denen an sich die vollständige Mobililisierung aller Arbeitskräfte und das Öffnen aller Grenzen gehören würde, gehen die wohlhabenden Staaten dazu über, die Grenzen zu schließen. Eine Weltwirtschaft, deren Zentren in Nordamerika, Europa und Japan sich in "Festungen" verwandeln und zugleich den Rest der Welt im Elend festhalten, statt Wohlstand um sich herum zu verbreiten, erweckt kein Vertrauen in zukünftige Stabilität. Verbal wird inzwischen von vielen gefordert, die Armut in den "verelendeten" Ländern zu beseitigen; aber konkrete und erfolgversprechende Projekte sind kaum zu erkennen. So wirksam die Ratschläge der neoliberalen Theorie in den Dimensionen der Betriebswirtschaft sind, so sehr versagen sie in volkswirtschaftlicher und vor allem in weltwirtschaftlicher Hinsicht. 


\subsection{Der "ökologische Hauptwiderspruch" der Marktwirtschaft}

Das Modell des "Wohlfahrtsstaats" hat, für sich betrachtet, durchaus eindrucksvolle Beweise seiner Leistungsfähigkeit gezeigt. Es gibt auch Beispiele dafür, daß unter günstigen Voraussetzungen einigen zunächst wenig industrialisierten Volkswirtschaften eine verspätete Industrialisierung gelungen ist, indem sie sich mit Hilfe von "Erziehungszöllen" vor der Konkurrenz der höher entwickelten Volkswirtschaften schützten. Neben den USA ist Deutschland ein Beispiel aus dem 19. Jahrhundert (Friedrich List). Nach dem zweiten Weltkrieg hat, auf Vorarbeiten beruhend, die bis vor die Meji-Restauration zurückreichen, Japan einen eindrucksvollen Aufstieg genommen. Neuerdings sind es vor allem die "vier kleinen Tiger", die den Anschluß an die westliche Form der Industrialisierung gefunden haben. Die neoliberale Theorie sieht darin den Beweis, daß es der Marktwirtschaft gelingt, armen Ländern zum wirtschaftlichen Aufstieg zu verhelfen. Auch die ökonomische "Öffnungspolitik" der Volksrepublik China orientiert sich an diesen Erfolgsmeldungen. Aber im Falle Chinas gibt es eine "kritische Grenze". In diesem Lande lebt etwa ein Fünftel der Menschheit. Diese extremen quantitativen Dimensionen zwingen China, nach anderen qualitativen Strukturen seiner Wirtschaft zu suchen. Wenn China das westliche "Erfolgsmodell" übernimmt, ohne es an Besonderheiten des Landes anzupassen, so könnte damit, gerade wenn es ökonomisch funktioniert, der Rubikon überschritten werden. Die Bedingungen der ökologischen Reproduktion des ganzen Planeten könnten erschüttert werden.

Ich will das an einem Beispiel erläutern. Gegenwärtig gibt es in der Welt etwa 400 Millionen Automobile, die etwa zu einem Drittel - neben je einem weiteren Drittel für Industrie und Haushaltungen - für die Belastung des Sauerstoffmantels der Erde mit Kohlendioxid und anderen Schadstoffen verantwortlich sind. Falls etwa, nach dem westlichen Vorbild, in absehbarer Zeit auch jeder zweite Chinese den "westlichen Lebensstandard" erreicht, für den nach unserem falschen Modell das Automobil als das entscheidende Statussymbol gilt, dann könnte, bei einer Verdoppelung des Automobilisierungsgrades, das Ökosystem der Erde zusammenbrechen. Wir nehmen dieses Beispiel nur als ein "Denkmodell", aber glauben, daß es sich lohnt, darüber nachzudenken, ob nicht wenigstens in China, wegen der extremen quantitativen Dimensionen, eine Alternative zu unserer parasitären "Verschwendungsökonomie" nötig wird und hier der Durchbruch zu einer "sozialistischen" Marktwirtschaft möglich sein könnte, zumal sie das offizielle Ziel des heutigen politischen Systems ist. Dabei sehe ich von den inneren Widersprüchen einer "Automobilgesellschaft" hier ab, namentlich dem gescheiterten Modell einer "autogerechten Stadt", die zur größten Stadtzerstörung der Geschichte geführt hat. Darüber mehr an anderer Stelle.

Selbst wenn man alle internen Widersprüche vernachlässigen würde, so macht das neoliberale Konzept eines ungesteuerten, planlosen Wirtschaftswachstums den "ökologischen Hauptwiderspruch" der kapitalistischen Wirtschaftsweise sichtbar. Wenn das Ökosystem 
des Planeten noch nicht gänzlich ruiniert ist, so verdankt das die kleine Minderheit der Weltbevölkerung in den hochindustrialisierten Staaten der großen Masse der Bewohner des Planeten, die von der Verschwendungsgesellschaft der westlichen Marktwirtschaft und von den Wohltaten unseres (unhaltbaren) Konzepts von "Modernisierung" noch ausgeschlossen ist. Eine Minderheit nutzt die Rohstoffe und Energiequellen des Planeten in rücksichtsloser Weise und ist für die exzessive Produktion von Schadstoffen verantwortlich. Unterstellt einmal, das Konzept der "Allianz für den Fortschritt" wäre nicht gescheitert und würden alle bisher von wirtschaftlicher "Entwicklung" ausgeschlossenen Länder in den Sog des heutigen "kapitalistischen Wohlfahrtsstaats" hineingezogen, so müßte das zur globalen Katastrophe führen. Die Menschheit würde sich durch einen "Ökozid" den Untergang bereiten.

Die Verallgemeinerungsfähigkeit ist der Prüfstein, an dem sich entscheidet, ob ein System Zukunftsperspektive hat oder nicht. Ich gehöre nicht zu den Zynikern, die geheim oder offen darauf hinarbeiten, die Masse der pauperisierten Menschen in den "unterentwickelten" Ländern im Zustand der Unterkomsumtion und der Verelendung zu halten, damit, auf ihrem Rücken, die Minderheit der Wohlhabenden in den "überentwickelten" Ländern ihren bisherigen parasitären Lebensstandard beibehalten kann. Als "Öko-Sozialist" bin ich vielmehr der Meinung, daß eine genaue Analyse der Situation zur gleichen Antwort führen muß, sowohl für die bereits industriell entwickelten Länder, wie für die Länder, die um ihre Industrialisierung noch kämpfen. Ich nehme an, daß eine ökologisch fundierte "sozialistische Marktwirtschaft" die Basis einer neuen, sozialistischen "Konvergenztheorie" sein könnte, und allein den Anforderungen gerecht würde, um verallgemeinert zu werden.

\subsection{Die Rolle der westlichen Arbeiterbewegung bei der Entstehung des "Wohlfahrts- staates"}

Auf die Entstehung des heutigen "Wohlfahrtsstaates", der Formen des permanenten Wirtschaftswachstums und der ökologisch unvertretbaren Verschwendungsökonomie haben auch die Arbeiterbewegungen und Gewerkschaften Westeuropas, Nordamerikas und Japans auf indirekte Weise Einfluß genommen. Aber es ist ihnen in keinem Fall gelungen, auf direktem Wege und in Übereinstimmung mit ihren eigenen Zielsetzungen die wirtschaftspolitischen Strukturen ihrer Länder zu prägen. Ihre mittelbare Wirkung ist jedoch beträchtlich. Um die ihm gefährliche sozialistische Kritik zu neutralisieren, ist der Kapitalismus dazu übergegangen, eine Ideologie der Partnerschaft von Kapital und Arbeit zu entwickeln, die früher vehement bekämpften Gewerkschaften in ein Element der Systemstabilisierung zu verwandeln und in unterschiedlichem Grade die Arbeiterschaft an den Erfolgen der hochindustrialisierten Wirtschaft teilnehmen lassen (bahnbrechend Henry Ford und Frederic Taylor). Dabei ist der Kapitalismus über sich hinausgewachsen. Massiv seit dem sog. "Korea-Boom", hat er die heutige phantastische und exzessive Konsumindu- 
strie aufgebaut (John Galbraith). Der westliche "Wohlfahrtsstaat" konnte auf diesem Wege viele Konflikte überbrücken.

Arbeiterbewegung und Gewerkschaften haben sich mit ihrer nur negativen Rolle zufrieden gegeben, das Wirtschaftssystem zu stimulieren und über sich hinauszutreiben, um auf dem Boden dieses Systems die Interessen der breiten Bevölkerung möglichst zu wahren, aber ohne das System selbst noch umbauen zu wollen. In einer Dualwirtschaft gibt es durchaus eine "Partnerschaft" von Kapital und Arbeit. Aber das heutige Problem liegt darin, daß die Arbeiterbewegung in diese Partnerschaft nicht mehr ihre spezifischen Interessen an einer nachhaltigen Gesellschaftsformation eingebracht hat. Inzwischen werden die Nachteile einer Form der Zusammenarbeit sichtbar, bei der die Arbeiterbewegung die Führung ganz und gar dem Kapital überließ und darauf verzichtete, ihre urspünglichen, sozialistischen Alternativvorstellungen weiterzuentwickeln. Diese "blinde" Partnerschaft beschränkte sich am Ende darauf, bei der Handhabung der Keynesianischen Wirtschaftspolitik die Instrumente der "Konjunktursteuerung" genauso gut, gelegentlich, wegen ihrer Querverbindungen zur Gewerkschaftsbewegung sogar etwas besser handhaben zu können. So konnten gelegentlich Regierungen unter Beteiligung sozialdemokratischer Parteien geradezu als "Krisenmanagement" dem Spätkapitalismus willkommene Dienste leisten.

Heute werden in ökologischer Sicht die Kosten deutlich, die daraus entstanden sind, daß es der Arbeiterbewegung nicht rechtzeitig gelungen ist, den Übergang zu alternativen Wirtschaftsstrukturen zu finden. Der Wohlfahrtsstaat, den der Kapitalismus aufgebaut hat, um den Herausforderungen durch die Arbeiterbewegung zu begegnen, hat zwar in Teilbereichen auch vernünftige Bedürfnisse der Bevölkerung befriedigt. Im ganzen hat dieser Weg aber zu einem selbstmörderischen "Krieg gegen die Natur" geführt (Hermann Scheer) und bedroht die menschliche Zivilisation mit dem Risiko ihres Untergangs.. Es waren nicht die Sozialdemokratischen Parteien und die Gewerkschaftsbewegung, die das ökologische Moment der Ausbeutung entdeckt hatten. Die neuen Anstöße kamen von ganz anderer Seite.

Als in Rußland die Oktoberrevolution ausbrach, haben die führenden deutschen Sozialdemokraten den Versuch bekämpft, den Sozialismus unter ökonomisch nicht entwickelten Bedingungen durchführen zu wollen. Sie beriefen sich auf Marx, der erklärt hatte, eine Gesellschaft müsse erst ihre Strukturen ganz zur Reife bringen, ehe ein Übergang zu einer höheren Gesellschaftsformation möglich sei. Es spricht viel für diese Annahme. Aber der verbreiteten Meinung, daß es 1917 für den Sozialismus in Rußland zu früh war, steht nun die andere Befürchtung gegenüber, daß ein Sozialismus, den die Menschheit vielleicht erst dann als Ausweg erkennen wird, wenn die Unhaltbarkeit der Widersprüche unserer Wirtschaftsweise ganz evident geworden ist, vielleicht zu spät kommt, weil die für eine Kurskorrektur benötigte Zeit möglicherweise nicht mehr ausreicht. Es wäre deshalb gut, wenn Karl Marx sich wenigstens an diesem Punkte geirrt hätte. Es spricht aber manches 
dafür, daß er gerade hier recht hatte. Der Zusammenstoß unserer Wirtschaftsordnung mit den ökologischen Realitäten scheint fast unabwendbar zu sein.

Das klingt nach einer Theorie des "Weltuntergangs". Oft ist eine Ende vorhergesagt worden, das dann doch nicht eintrat. Eine düstere Perspektive kann auf Denkfehlern beruhen. Sie muß selbstkritisch und gründlich überprüft werden. Aber es gibt zahlreiche historische Erfahrungen, wie stabil erscheinende Systeme über Nacht von der Bildfläche verschwanden. Gerade der Zusammenbruch des sowjetischen Blocks zeigt ein solches Beispiel. Ganz allgemein gilt, daß jedes System unweigerlich zusammenbricht, sobald die Bedingungen seiner Reproduktion nicht mehr gegeben sind. Die großen Reiche des Altertums waren "Kolosse aus Erz", die aber auf "tönernen Füßen" standen. Der Untergang des Römischen Weltreichs, das mit seiner pax romana eine definitive Weltordnung sein wollte, brach zusammen, nachdem es sich "zu Tode gesiegt" hatte. Dieser tiefe Einschnitt in der historischen Entwicklung Europas hat viele Generationen verstört. Manchmal gibt es ganz einfache Ursachenverkettungen. So verfielen große Seemächte, als sie die Wälder zerstört hatten, die sie für den Schiffsbau brauchten. Es scheint daher nicht unvernünftig, sondern muß als ein Zeichen von Nüchternheit gelten, mit der Möglichkeit zu rechnen, daß sich die menschliche Zivilisation wegen ihrer atavistischen Produktionsweise, die sie nicht mehr beherrscht, selber ihren Untergang bereitet. Vieles spricht dafür, als würde der system-interne Zwang unseres Wirtschaftssystems zum permanenten Wachstum, der für die kapitalistische Marktwirtschaft charakteristisch ist, die ökologischen Grundlagen des ökonomischen Systems untergraben. Irrational und zynisch wäre es, die schlechten Nachrichten zu verdrängen.

Hier wird im übrigen aber auch keine Unheilsprophezeihung ausgesprochen, sondern nach Auswegen gesucht. Es gibt vielleicht doch Möglichkeiten, die Katastrophe abzuwenden. Ohne jeden Dogmatismus könnte eine "sozialistische Marktwirtschaft" an das theoretische Interesse von Marx und Engels anknüpf en. Sie überwanden den "utopischen" Sozialismus, um erst einmal die Bewegungsgesetze der kapitalistischen Entwicklung zu erfassen. Aber ihr Ziel war es, die Errungenschaften des bürgerlichen Zeitalters dadurch zu erhalten, daß sie den bürgerlichen Horizont überschritten, um nach einem vernünftigen "Metabolismus" zwischen Mensch und Natur zu suchen. In ähnlicher Weise müßten wir heute, gestützt auf ganz neue Erkenntnisse, die wissenschaftlichen, technischen, politischen und rechtlichen Errungenschaften der entwickelten bürgerlichen Gesellschaft erfassen, um sie im Rahmen eines Dualsystems, nach dem Gegenstromprinzip, den vernünftigen menschlichen Bedürfnissen unterzuordnen, um sie dadurch dauerhaft zu erhalten. Schließlich entspricht es wohl einem Hauptbedürfnis der menschlichen Zivilisation, sich nicht selbst den eigenen öklogischen Ast abzusägen. 


\section{3. Ökonomie und Ökologie im Lichte der Entropie-Theorie}

Das vorherrschende Weltwirtschaftssystem zeigt sich in ökologischer Hinsicht destruktiv. Das wird besonders deutlich, wenn wir die vorherrschenden Formen des ungesteuerten, nur von den Prämissen der Kapitalverwertung abhängigen Wirtschaftswachstums unter dem Gesichtspunkt des Energieverbrauchs im Lichte der Theorie von der Entropie im Sinne der beiden Hauptsätze der Thermodynamik betrachten. Eine solche Sichtweise mag auf den ersten Blick übermäßig theoretisch klingen. Aber beim zweiten Blick hilft der Umweg über dieses theoretische Fundament, um ganz praktische Fragen der politischen und wirtschaftlichen Orientierung einschätzen und entscheiden zu können.

Gegen Ende des des 19. Jahrhunderts sind die Prozesse der Energieumwandlung gründlich erforscht worden. Aber im Gefühl, daß die an den Umwandlungsprozessen beteiligten Naturressourcen praktisch unbegrenzt seien, hat die Wirtschaftswissenschaft bis vor kurzem diesen Fragen keinerlei Bedeutung beigemessen. Erst in der heutigen Diskussion um das Verhältnis von Ökonomie und Ökologie wird die Entropie-Problematik wieder erfaßt (Nicholas Georgescu-Roegen, Herman Daly, Barry Commoner, Jeremy Rifkin, Juan Martínez Alier, Hermann Scheer u.a.).

\subsection{Die Bedeutung der "Entropie"-Theorie für die Volkswirtschaft}

Der Erste Hauptsatz der Thermodynamik lautet, daß Energie eine Naturkonstante ist, die weder geschaffen noch zerstört, sondern nur umgewandelt werden kann. Wenn man ein Stück Kohle verbrennt, geht keine Energie verloren, sie wandelt sich nur in andere, gasförmige Verbindungen, in Wärme und in Reststoffe um. Die ursprüngliche, in der Kohle verkörperte Energie hat sich quantitativ nicht vermindert; die Energiemenge bleibt konstant. Es hat sich nur die Erscheinungsform der Energie verwandelt. Für unsere Fragen ist aber entscheidend der Zweite Thermodynamische Hauptsatz. Er besagt, daß der Prozeß der Energieumwandlung nicht umkehrbar ist. Der Energiefluß findet nur in einer Richtung statt; er verläuft von einem wärmeren zu einem kälteren Körper. Dabei wird aus der "verfügbaren" Energie "unverfügbare". Die durch Verbrennung der Kohle entstandene Wärme, die neuen chemischen Verbindungen und die Rückstände lassen sich nicht wieder in das Stück Kohle zurückverwandeln. Es kann kein perpetuum mobile geben, Arbeit und Bewegung sind nur möglich, wenn Energie eingesetzt wird.

Heute erscheinen diese Sätze jedem plausibel. Albert Einstein, der das traditionelle physikalische Weltbild in seinen Fundamenten erschüttert hatte, hielt diese beiden Hauptsätze der Thermodynamik für unwiderleglich. Aber es war mühevoll und dramatisch, die Richtigkeit dieser Erfahrungssätze theoretisch zu beweisen. Für die Unumkehrbarkeit dieser Prozesse bei der Energieumwandlung hat 1868 der Physiker Rudolf Clausius den Begriff der "Entropie" eingeführt. Wenn bei der Umwandlung von verfügbarer Energie nichtverfügbare Energie entsteht, so nimmt dabei, wie er formulierte, die "Entropie" zu. 
Damit wollte er ausdrücken, daß Energie mit einem hohem Ordnungsgrad zu einer Energieform von geringerem Ordnungsgrad "degradiert" wird. Stark abgekürzt formuliert kann man die dabei entstehenden, nicht mehr verfügbaren Endprodukte der Energieumwandlung als "Abfall" bezeichnen. Nur teilweise und nur unter Verwendung neuer Energiequellen läßt sich Abfall im Stoffkreislauf wiederverwenden. Das Recycling löst das Entropie-Problem nicht, auch wenn es vernünftig ist, Rohstoffe mehrfach zu verwerten. Aber entscheidend für die Bewertung des Recycling ist der hierfür erforderliche Bedarf an neuer Energie.

Das Entropieproblem wird von äußeren Rahmenbedingungen modifiziert. Es gilt im strengen Sinne nur in "geschlossenen Systemen". Im Blick auf die Rohstoffe ist die Erde ein geschlossenes System. Hier gibt es keinen, oder doch nur einen vernachlässigenswert geringen Austausch mit anderen Systemen des Universums. Der gelegentliche Einschlag eines Meteoriten spielt für die menschliche Wirtschaftsordnung keine Rolle. Bei der Produktion von Gebrauchsgütern erschöpfen wir die vorhandenen Ressourcen auf eine irreversible Weise. Eine solche theoretisch richtige Erkenntnis könnte allerdings dann praktisch belanglos sein, wenn diese Ressourcen quantitativ für die begrenzten Bedürfnisse der menschlichen Ziele ausreichen würden. Aber inzwischen sind wir uns bewußt geworden, daß die Vorräte an Naturschätzen nicht nur "prinzipiell" endlich sind. Sie sind inzwischen auch "praktisch" knapp geworden. Das gilt vor allem für die fossilen Energiequellen und einige seltene Metalle. Jede Produktion ist begriffsnotwendig mit der Produktion von Abfall verbunden. Auch das könnten wir theoretisch akzeptieren, aber praktisch vernachlässigen, wenn nicht die Bürgermeister aller unserer Gemeinden vor der politischen Frage stünden, wie sie den Abfall entsorgen sollen, in dem wir bereits zu ersticken drohen.

Aber in einer anderen Hinsicht ist die Erde kein geschlossenes, sondern ein offenes System. Es gibt energetische Prozesse, bei denen keine "Degradierung" stattfindet: Die der Erde aus dem Kosmos zufließende Sonnenenergie bringt im Wege der Photosynthese pflanzliches, tierisches und menschliches Leben hervor. Diese biologischen Prozesse auf unserem Planeten kehren den entropischen Prozeß um. Die Photosynthese wurde in dieser abstrakten Sprache als "negative Entropie" bezeichnet. Es würde uns allerdings mehr einleuchten, wenn wir dafür einen positiven Begriff verwenden würden. Statt die Energie im Prozeß ihrer Umwandlung zu "degradieren", bringen die biologischen Lebensprozesse auf unserem Planeten neue und höhere Ordnungsstrukturen hervor; "Leben" aber ist kein Abfall. Hierin liegt die große Chance der Solarenergie für die menschliche Zivilisation. Es ist eine merkwürdige Tatsache, daß die moderne Wirtschaftswissenschaft, die alle Faktoren des ökonomischen Prozesses aufs Genaueste untersucht hat, bis heute das Abfallproblem, das mit jedem Produktionsprozeß begriffsnotwendig verbunden ist, auf der Höhe der Entropie-Theorie nicht gestellt und bis vor kurzem einfach verdrängt hat. Dafür gibt es einige Gründe. Früher konnte man es sich einfach nicht vorstellen, auch wenn man es wußte oder doch hätte wissen können, daß die Ressourcen der Natur so begrenzt sind, wie 
wir das inzwischen erfassen. Das theoretische Problem der "Entropie" erschien auch deshalb nicht als praktisch bedeutsam, weil es so hochgegriffen hatte, daß es für die realen Zwecke der Wirtschaftswissenschaft nahezu unfaßbar wurde. Es ging bei der Diskussion um die Hauptsätze der Thermodynamik nur am Rande um Probleme des Planeten Erde; nicht einmal unser Sonnensystem war der letzte Bezugsrahmen. Die damaligen Forscher waren mit dem ganzen uns bekannten Universum beschäftigt. Vielleicht würden die atomaren Prozesse unserer Sonne in irgend einer Zukunft einmal enden und mit ihnen auch das Leben auf der Erde. Auf dieser Abstraktionshöhe kannte man auch keine "nicht erneuerbaren" Ressourcen in unserem jetzigen Sprachgebrauch. Denn bekanntlich sind die fossilen Rohstoffe, die wir heute als "nicht erneuerbar" bezeichnen, wenn auch in historisch nicht vorstellbaren Zeiträumen, ihrerseits im Wege der Photosynthese entstanden. Um das Entropie-Problem für die praktischen Fragen der Wirtschaftswissenschaft faßbar zu machen, mußte erst die Begrenztheit der Naturressourcen fühlbar werden. Für die praktischen Bedürfnisse der Volkswirtschaft betrachten wir als "nicht-erneuerbar" heute solche Rohstoffe und Energiequellen, die nicht in kalkulierbaren "Umtriebszeiten" nachwachsen.

\subsection{Der "energetische Imperativ" und die marxistische Theorie}

Zu Beginn dieses Jahrhunderts stellte der Chemiker Wilhelm Ostwald in Anlehnung an Immanuel Kant einen "energetischen Imperativ" auf und formulierte: "Vergeude keine Energie, verwerte sie". Diese Haltung war Ausdruck der Entropie-Debatte der Zeit. Sie war im übrigen belastet mit unhaltbaren Prämissen eines philosophischen "Monismus", der alle Kulturerscheinungen "monokausal" auf energetische Prozesse zurückführen wollte, worüber Max Weber die Schalen seines Spotts ausgegossen hat (Energetische Kulturtheorien, 1909). Das heutige Problem der praktischen Begrenztheit der nicht erneuerbaren Ressourcen und die Belastung der menschlichen Umwelt durch die Schadstoffe, die bei den Verwertungsprozessen freigesetzt werden, hatte Ostwald noch nicht im Auge. Er beschränkte sich auf die Forderung, mit der Energieumwandlung, die unweigerlich eine "Degradierung" des Energieflusses mit sich bringt, rational umzugehen und den größtmöglichen Nutzen aus den vorhandenen Ressourcen an Materie und Energie für die Menschheit zu ziehen. Das war vernünftig, aber für unsere heutigen Fragestellungen nicht genug.

Marx und Engels befaßten sich ebenfalls eingehend mit dem Entropie-Problem, aber auch sie erkannten seine heutige praktische Relevanz noch nicht. Ihre Aufmerksamkeit war ganz davon gefesselt, daß die Gesellschaft einen früher nicht vorstellbaren Reichtum produzierte, aber wegen innerer Widersprüche ihr eigenes Potential nicht realisieren konnte und gleichzeitig die Lebensbedürfnisse der unmittelbaren Produzenten grob mißachtete. Sie konnten nicht voraussehen, wie der Kapitalismus auf ihre radikale Kritik reagierte, und, um seinen Untergang abzuwenden, ein System der Verschwendungsökonomie errichtete, das zwar den unmittelbaren Produzenten zum Teil zu Gute kam, 
zugleich aber die Lebensbedingungen der Menschheit insgesamt bedrohte. Das erklärt zum Teil, warum es ihnen noch nicht möglich war, ihre Theorie der Ausbeutung zu der letzten Konsequenz weiterzuentwickeln, bis der Hauptwiderspruch der kapitalistischen Wirtschaft sichtbar wurde, der in der Belastung der Umwelt und der Zerstörung der früher für unerschöpflich gehaltenen Ressourcen liegt, - dem Wasser der Flüsse und der Weltmeere, den Wäldern, dem fruchtbaren Ackerboden und sogar der Lufthülle des Planeten. Dieser ökologische "Hauptwiderspruch" der industriellen Produktion ist ihnen vielleicht aber auch deshalb entgangen, weil die Theorie der Entropie mit ihren philosophischen Prämissen nicht gut übereinstimmte. Engels hat die Arbeiten des Entropie-Theoretikers Clausius mit größtem Interesse verfolgt. Um die Größe des Jahrhunderts zu beschreiben hat er gelegentlich geradezu auch vom "Jahrhundert von Clausius" gesprochen. Aber aus Gründen ihrer philosophischen Grundannahmen konnten weder er noch Marx sich mit dem Gedanken der Entropie wirklich anfreunden. Engels meinte, Clausius sei es nur gelungen, das Problem zu stellen, noch nicht aber, es auch zu lösen. Er zählte darauf, daß es eines Tages doch möglich sein werde, nicht nur für das relativ offene System des Planeten Erde, sondern auch für das Universum als ganzes, die Umkehrbarkeit des Energieflusses und damit die schließliche die Aufhebung des Entropie-Theorems zu beweisen. So hat wohl auch ihre Voreingenommenheit dazu beigetragen, daß in der marxistischen Theorie die Ökologie zum blinden Fleck werden konnte.

Es wäre überheblich, Marx und Engels daraus einen Vorwurf zu machen. Uns stehen heute ganz andere Daten zur Verfügung als den Forschern des 19. Jahrhunderts. Aber weil auch die nach ihnen kommenden Generationen von Chemikern, Physikern und Ökonomen in den sozialistischen und kapitalistischen Staaten sich dieser Thematik nicht oder nicht radikal genug zugewandt haben, hat sich diese ökologisch blinde Stelle in der Theorie des Marxismus auf entsetzliche Weise ausgewirkt. Fünf Mio km ${ }^{2}$, ein Viertel der ehemaligen UdSSR, damals des größten Landes der Erde, sind heute ökologisch schwer belastet. Es ist bekannt, mit welcher Arroganz der Macht auch die Chef-Ökonomen der DDR alle abgebürstet haben, die auch nur die geringste Kritik an den ökologischen Verwüstungen der DDR zu äußern gewagt hatten. Obwohl Marx und Engels bei der damaligen geringeren Weltbevölkerung und vor dem Beginn der heutigen Verschwendungsökonomie die reale Begrenztheit der Ressourcen nicht erfaßt haben und wohl auch noch nicht erfassen konnten, hatten sie aber immerhin bereits einen klaren Blick dafür, daß es darum geht, die erstaunliche Entfesselung der Produktionskräfte der kapitalistischen Wirtschaftsweise zu beenden und sie den menschlichen Bedürfnissen unterzuordnen. Es finden sich bei ihnen eine Reihe sensibler und weitsichtiger Bemerkungen. So warnt Engels in der Dialektik der Natur vor der Illusion, als könnten die Menschen lernen, die Natur zu "beherrschen": Für jeden Sieg "rächt sie sich an uns". Er verurteilt es, mit der Natur so umzugehen, "wie ein Eroberer ein fremdes Volk beherrscht"; das Ziel müßte vielmehr sein, daß sich die Menschen wieder als "Eins mit der Natur fühlen". Der "Gigantismus" des sowjetischen Modells von Sozialismus kann sich kaum auf Marx und Engels berufen. Auch 
Marx spricht davon, daß es die Aufgabe der Menschen ist, einen vernünftigen "Stoffwechsel" oder "Metabolismus" mit der Natur wiederherzustellen. Die Menschen sind nicht "Eigentümer" der Erde, sondern bloß "Nutznießer" und haben die Verantwortung, sie der Nachwelt "in gutem Zustand zu übergeben".

Erst heute können wir ganz ermessen, daß die kapitalistische Ausbeutung nicht darauf beschränkt war, die menschlichen Interessen der unmittelbaren Produzenten zu verletzen; sie ist auch tatsächlich dabei, wie Marx voraussah, die Menschen im Produktionsprozess "überflüssig" zu machen, worin er die Quelle revolutionärer Bewegungen sah. Heute können wir darüber hinaus erkennen, daß die Selbstreproduktion des Kapitals, die alle Investitionen an die Zinsrate knüpft und zu ständig wachsender Produktion auf immer höherer Stufenleiter gezwungen ist, den "Krieg gegen die Natur", der mit der neolithischen Revolution begann, derart gesteigert hat, daß die Lebensbedingungen der menschlichen Zivilisation als solche gefährdet sind.

\subsection{Neudefinition von Sozialismus}

Die "soziale" Marktwirtschaft und der kapitalistische "Wohlfahrtsstaat" suchen nach Möglichkeiten, die Eigengesetzlichkeiten des Marktes auch für die Interessen der Bevölkerung zu nutzen. Das Gesamtsystem wird aber nach wie vor von den Reproduktionsbedingungen der Kapitalverzinsung beherrscht. Demgegenüber müßte eine "sozialistische" Marktwirtschaft das Marktgeschehen den Bedingungen einer dauerhaften und nachhaltigen Wirtschaftsweise des Gesamtsystems unterordnen. Ohne das Dualsystem aufzuheben, bei dem öffentliche Hand und Markt in relativer Autonomie nebeneinander bestehen, käme hier dem öffentlichen Sektor die gegenüber der nur "sozialen" Marktwirtschaft quantitativ und qualitativ erhöhte Aufgabe zu, dem Markt deutlich Ziele und Rahmenbedingungen vorzugeben.

Um Mißverständnisse zu vermeiden, möchte ich einige Begriffe erläutern. Gelegentlich kritisiere ich hier das Prinzip der "Profitmaximierung". Diese Kritik muß aber richtig begrenzt werden. Einem Kaufmann oder einem Unternehmer kann kein Vorwurf daraus gemacht werden, wenn er einen Preis für Waren verlangt, der über den Kosten liegt, zu denen er eingekauft oder produziert hat. Es versteht sich von selbst, daß jedes Wirtschaften unmöglich würde, wenn die Ausgaben die Einnahmen übersteigen. Das gilt nicht nur für die kapitalistische Wirtschaft, sonder ist genau so wahr bei "gemeinnützigen" Unternehmen und auch dem Staat selber, und zwar unabhängig davon ob es der Staat einer kapitalistischen oder einer sozialistischen Gesellschaft ist. Auch ein privater Haushalt bricht zusammen, wenn jemand mehr ausgibt, als er einnimmt. Gegen das Prinzip der "Gewinnmaximierung" wird hier kein Einwand erhoben, soweit und solange die Gesetzmäßigkeiten des Marktes funktionieren und die wirtschaftliche Entwicklung dem rudimentären Marktprinzip anvertraut werden kann, daß dort am meisten investiert wird, wo die größten Gewinne erwartet werden. Sof ern Marktbedingungen herrschen, werden auch 
andere Anbieter von den lockenden Gewinnen angezogen, was über den Mechanismus des Wettbewerbs die Preise drückt und, wie es Marx formuliert, zur "Verwohlfeilerung" der Waren führt. Wir beobachten zum Beispiel auf dem Computer-Markt, wie dort, wo solcher Wettbewerb herrscht, die Produkte immer besser und immer billiger werden. Aber diese Marktmechanismen funktionieren nicht immer und überall, auch nicht in allen Bereichen der Computer-Industrie. Bereits faktische Monopolstellungen können diese Gesetzmäßigkeiten einengen oder aufheben.

Geld spielt auch in der Öffentlichen Verwaltung eine entscheidende Rolle. Die Einkünfte stammen in der Hauptsache aus allgemeinen Steuern, Beiträgen und Gebühren. Es ist nicht möglich, den Wert für gewisse administrative Dienste in Geld zu bestimmen. In diesem Sinne haben eine "Rechtsordnung", ein System sozialer Sicherheit, die Sicherheit und Ordnung, die die "Polizei" verbürgen soll, ein Bildungssystem, die kulturellen und sozialen Dienstleistungen einer Gesellschaft u.v.a.m. keinen "Preis". Aber die Fonds, aus denen die Ausgaben für diese öffentlichen Funktionen finanziert werden, müssen nicht nur "sparsam" und "wirtschaftlich" eingesetzt werden; sie müssen auch über "Wertschöpfungsprozesse" erst "erwirtschaftet" werden. Ohne daß die Verwaltung ihrerseits an der Profitmaximierung teilnimmt, ist es sinnvoll, wenn sie sich bei dem Einsatz der knappen Mittel an Methoden des Managements der privaten Wirtschaft orientiert und sorgfältige Kosten-Nutzen-Analysen anstellt.

Wenn die öffentliche Verwaltung aber nicht administrative Entscheidungen produziert, sondern direkt "unternehmerisch" tätig wird, wie das bei den "öffentlichen Unternehmen" des Staates und der Gemeinden der Fall ist, können diese Tätigkeiten auch unmittelbar "kaufmännisch" ausgestaltet werden. Dabei werden die öffentlichen Ziele dieser Unternehmen durch Gesetze, Gemeindesatzungen und Gesellschaftsverträge festgelegt. Seit den wegweisenden Reformen der 20er Jahre werden solche Bereiche der Verwaltung aus dem öffentlichen Haushalt mit seiner "kameralistischen" Einnahmen- und Ausgabenrechnung ausgegliedert und als "Sondervermögen" behandelt, wobei die Unternehmen in den Formen von öffentlichen "Eigenbetrieben" einer kaufmännischen Geschäftsführung und einer echten Gewinn- und Verlustrechnung unterworfen worden sind. Auch bei "öffentlichen Unternehmen" verlangen die knappen Mittel sorgfältige wirtschaftliche Kalkulation. Aber solche Unternehmen unterliegen nicht dem Prinzip der Profitmaximierung. Wasserbetriebe, Unternehmen der Abfallentsorgung und des öffentlichen Personennahverkehrs wachsen nicht weiter, sobald der öffentliche Bedarf gedeckt ist. Solange die Eisenbahnen ihren Personenverkehr in Mischfinanzierung auch aus dem Güterverkehr mitfinanzieren konnten, der bis in die 50er Jahre überwiegend auf der Schiene abgewickelt wurde, und solange der öffentliche Personennahverkehr noch nicht durch den Individualverkehr ruiniert worden ist, haben diese öffentlichen Verkehrsunternehmen auch Gewinne erwirtschaftet. Auch bei "gemeinnütziger" Tätigkeit entstehen "Kosten". Sie werden durch Umlagen aufgebracht oder aus bestimmten Fonds zur Verfügung gestellt oder aus "Gewinnen" finanziert. Nach dem Gemeinnützigkeitsgesetz darf nur eine geringe Verzin- 
sung der Gewinne an die Gesellschafter ausgezahlt werden. Wenn der Gewinn diese Ausschüttungsrate übersteigt, wird er reinvestiert oder, wenn die Organisation eng an die öffentliche Verwaltung gebunden ist, an den öffentlichen Haushalt abgeführt.

Bei den Eigenbetrieben unterscheiden sich im Prinzip weder Management noch Kalkulation oder Rechnungswesen prinzipiell von denen privater Unternehmen. Es ist eine Tatsache, daß die Eigenbetriebe, wie sie in den 20er Jahren entwickelt worden sind, gründlicher Reformen bedurften. Aber eine Privatisierung in der Form der Aktiengesellschaft wird den öffentlichen Aufgaben nicht gerecht, weil der Vorstand auf das Wohl des "Unternehmens", nicht auf das der "Unternehmer" und ihrer öffentlichen Ziele verpflichtet ist. Auch eine bloß "formelle Privatisierung", die die Geschäftsanteile vollständig in der öffentlichen Hand beläßt, geht im Falle der Aktiengesellschaft einen Schritt zu weit. Es ist zu beklagen, daß Sozialdemokratie und Gewerkschaften dem Privatisierungsdruck nachgegeben haben, statt moderne Reformkonzepte für die Struktur der öffentlichen Unternehmen zu entwickeln.

Auch wenn öffentliche Unternehmen als Instrumente einer "Bedarfsdeckungswirtschaft" in der Regel nach dem Prinzip der "Kostendeckung" arbeiten, kann es nicht als unzulässig betrachtet werden, daß dabei Gewinne entstehen. In einer Zeit, in der öffentliche Haushaltsmittel knapp geworden sind, ist es für die Bürger von großem Interesse, daß die öffentliche Verwaltung im Rahmen des "Unternehmerstaats" einen Teil ihrer Leistungen auch durch eigene Einnahmen finanzieren kann. Das entlastet den "Steuerstaat" und damit auch den Steuerzahler. In der Dualwirtschaft trägt der öffentliche Wirtschaftssektor den Charakter einer Wirtschaft der "Bedarfsdeckung" und damit der "einfachen Reproduktion". Er kann sich dabei in seinem "Management" aber in seinen Zielsetzungen dem Sektor der marktorientierten Profwirtschaft anpassen. Damit klingt eine Kritik am Prinzip der Profitmaximierung an. Aber diese Kritik beschränkt sich darauf, dem Marktsektor mit dem ihm inhärenten Profitprinzip einen Sektor öffentlicher Inf rastrukturen gegenüberzusetzen, um Marktversagen auszugleichen. Der brasilianische Ökologe Jorge Lutzenberger hat mit Recht darauf verwiesen, daß alle biologischen Wachstumsprozesse ein negatives feed-back kennen: Wenn eine vernünftige Grenze erreicht ist, müßte das Wachstum eigentlich aufhören oder die Wachstumskurve sich doch abflachen. Es zeichnet sich ab, da $\beta$ in einigen Sektoren unserer Wirtschaft Grenzen eines sinnvollen Wirtschaftswachstums erreicht oder schon überschritten sind. Das würde aber nicht bedeuten, daß dann die Welt zusammenbricht, da dann immer noch die jahrtausendealten Techniken der "einfachen Reproduktion" zur Verfügung stehen, wobei der öffentliche Sektor ein Modell darstellt.

Aber auch dort, wo das Prinzip der "Profitmaximierung" bekämpft werden muß, weil es keine vernünftige Funktion mehr erfüllt, heißt das nicht, daß der "öffentliche Sektor" der Wirtschaft, der den Markt begrenzt, seinerseits an die Stelle einer strengen Kostenkalkulation Gleichgültigkeit treten lassen darf, wie die doch entstehenden Kosten aufgebracht 
und die Mittel eingesetzt werden. Auch hier sind Wirtschaftsprinzipien einer genauen, kaufmännischen Kalkulation anzuwenden. Der Sozialismus des UdSSR-Modells ist auch deshalb zusammengebrochen, weil der Irrglaube entstanden war, als hätte man die bürgerliche Gesellschaft ganz überschritten und könne sorglos "aus dem Vollen" wirtschaften. Indem eine "sozialistische" Marktwirtschaft definitionsgemäß auf eine "Vollsozialisierung" verzichtet, trägt sie einem Einwand Rechnung. Eine sozialistische Wirtschaft hat Schwierigkeiten, das Problem einer rationalen und sparsamen Wirtschaftsrechnung zu lösen, wie das in den 20er Jahren namentlich Ludwig v. Mises begründet hat. Aber daraus folgt in keine Weise, auf eine "Dualwirtschaft" zu verzichten, wo sie erforderlich ist. Der öffentliche Sektor muß sich jedoch bei seinem Wirtschaftsgebahren am privaten Sektor orientieren, wie ein effizientes Management aufgebaut und mit knappen Mitteln "sparsam" und "wirtschaftlich" umgegangen werden kann, aber ohne dessen Ziele - die Profitmaximierung - mitzuübernehmen.

Joseph Schumpeter hielt eine sozialistische Gesellschaft für erforderlich und auch für gangbar, sobald auch die kapitalistische Wirtschaft ihre historische Funktion erfüllt haben würde. Die ökologische Problematik verlagert diesen damals noch weit entfernten Zeithorizont nach vorn in unsere Gegenwart. Aber Schumpeter hat immer betont, daß Kapitalismus und Marktwirtschaft im Einsatz von Arbeitskraft, Kapital und Rohstoffen nicht übertroffen werden können. Es gibt keine "sozialistische Produktionsweise", die der kapitalistischen überlegen sein könnte. In dem Konzept einer "sozialistischen Marktwirtschaft" muß sich daher der öffentliche Sektor im eigenen Interesse an Management und Arbeitsorganisation des privaten Sektors orientieren, der eine rationale Form der Produktivität entwickelt, wie es eine sozialistische Wirtschaft von sich aus niemals könnte. Der Kapitalismus scheiterte nach der Auffassung von Schumpeter nicht an seinen Schwächen, sondern an seinen Stärken. Er macht sich wegen der ständigen Steigerung der Arbeitsproduktivität am Ende überflüssig. Dementsprechend bestand für ihn Sozialismus nicht darin, eine neue und höhere Form der Arbeitsproduktivität zu entwickeln, um den Kapitalismus zu überholen, sondern umgekehrt darin, sich dort von der Profitwirtschaft abzuwenden, wo sie ihre Aufgabe der Innovation erfüllt, ihre soziale Funktion verloren und kontraproduktiven Charakter angenommen hatte. Da die gesellschaftlichen Entwicklungen asynchron laufen, ist das für einige Segmente der Wirtschaft, nicht aber für den gesamten ökonomischen Prozeß, bereits jetzt der Fall.

Schumpeter stellte sich vor, daß, nach einer gewissen Sättigung vernünftiger Bedürfnisse, die Menschen sich anderen Zielen zuwenden würden, die faszinierender sind als der Kampf um immer mehr Gebrauchsgüter, unabhängig davon, ob sie die Lebensqualität wirklich erhöhen oder auch nicht. Auch Keynes war davon überzeugt, daß das Wirtschaftswachstum einmal zu einem gewissen Ende kommen werde. Beide waren deshalb an den kulturellen Dimensionen der Gesellschaft stark interessiert, weil sich in ihnen die Alternativen zur reinen Wirtschaftsgesellschaft bilden müßten. Das stand in Einklang mit den Vorstellungen von Marx, der immer nach Qualitäten Ausschau hielt, die für den homo 
sapiens charakteristischer sind als die Befriedigung seiner primären Bedürfnisse. In dieser Perspektive erschien es in der Vergangenheit immer als eine der wichtigsten Funktionen des "Gemeindesozialismus", ein kulturelles Ambiente zu schaffen, das jenseits der Arbeitswelt Chancen der persönlichen Entfaltung eröffnet, in der Tiefe wie in der Breite (Kommunale Museen, Theater, Opernhäuser, Orchester, Musikschulen, Volkshochschulen, Schultheater, bezahlbare Eintrittskarten u.v.a.m.). Der Vergleich mit anderen Ländern macht den Reichtum einer kommunalen Kulturlandschaft sichtbar, die auf solchen "gemeindesozialistischen Traditionen" beruht. Deshalb sind es schlimme Signale des Neoliberalismus, wenn aus ökonomischen Gründen kulturelle Strukturen preisgegeben werden, statt ihre Finanzierung an neue Gegebenheiten anzupassen. Als ein solches negatives Signal ist in Berlin die Schließung des Schillertheaters verstanden worden, die gezeigt hat, daß in der heutigen Sozialdemokratie die alten "munizipalsozialistischen" Traditionen, mit denen sie groß geworden ist, nicht mehr am Leben sind. Auch der Abriß des alten Theaters der Peking-Oper in der Goldfischgasse von Beijing zu Gunsten eines Warenhauses gehört in diese Linie.

Um eine "sozialistische Marktwirtschaft" aufzubauen, genügt es nicht, Wirtschaftsmacht in öffentlicher Hand zu behalten. Der öffentliche Sektor muß auch eine andere Wirtschaftspolitik treiben als der Sektor der Profitmaximierung. Wenn früher der öffentliche Sektor als "Staatssozialismus" und als "Gemeindesozialismus" bezeichnet wurde, so bestand immerhin der richtige Kern dieser Begriffe darin, daß dieser Sektor selbst nicht unmittelbar der "Profitmaximierung" dient, sondern "Bedarfsdeckungswirtschaft" betreibt, um unter sparsamer und wirtschaftlicher Verwendung knapper finanzieller Ressourcen eine nachhaltige Wirtschaftsweise des Gesamtsystems zu gewährleisten und damit "öffentliche Interessen" sicherzustellen. Wenn der öffentliche Sektor ganz in den Sog der Profitmaximierung gezogen wird und die öffentliche Hand ihre Wirtschaftsmacht preisgibt, indem sie ihr Tafelsilber verhökert, um ein Abendbrot zu bezahlen, öffentliche Unternehmen privatisiert und kommunalen Grundbesitz veräußert, den sie in den Jahrzehnten ihrer Bodenreformpolitik erworben hat, um ein momentantes Haushaltsloch zu stopfen, dann verliert er seine Fähigkeit, nach dem Gegenstromprinzip ein vernünftiges Gegengewicht zum Marktsektor zu bilden. Im Lichte der inzwischen verfügbaren Daten muß es dem öffentlichen Sektor und den Entscheidungen des politischen Systems auf der Ebene des Gesamtstaats oder der der Gemeinden überlassen werden, was für eine nachhaltige, auf Dauer reproduktionsfähige Form der menschlichen Zivilisation erforderlich ist. Bei Strafe des Zusammenbruchs unserer Zivilisation muß die 200-jährige Periode einer blindwütigen Entfesselung aller Produktivkräfte beendet werden. Das Ziel der wirtschaftlichen Entwicklung muß nun darauf gerichtet werden, die fabelhaften neuen Produktivkräfte endlich den menschlichen Bedürfnissen unterzuordnen und einen vernünftigen Stoffwechsel zwischen Mensch und Natur wiederherzustellen.

Das führt zu einigen Schwierigkeiten zwischen Ländern, die bereits ausreichend industriell entwickelt sind, und solchen Ländern, die dabei sind, eine industrielle Basis der 
gesellschaftlichen Modernisierung und zur Beseitigung der Armut erst zu schaffen. Aber auf der anderen Seite ergeben sich bei dieser Betrachtungsweise auch einige interessante Perspektiven. Es könnte sehr nützlich für Länder sein, die jetzt ihr gesellschaftliches System neu aufbauen, die Probleme zu berücksichtigen, in die die "überentwickelten" Länder geraten sind. Es könnte dabei eine falsche und bedauerliche Investition knapper Devisen verhindert werden, wenn Wege vermieden würden, die sich bereits anderswo als Irrwege herausgestellt haben. Gleichzeitig bietet diese "öko-sozialistische" Sicht auch die Perspektiven einer neuartigen "sozialistischen Konvergenztheorie", die es möglich machen könnte, das sozialistische Denken in den westlichen Ländern langf ristig wieder zu beleben und damit neue Akzente in die internationale Zusammenarbeit zu bringen.

Im Gegensatz zu denen, die glauben, daß nach dem Zusammenbruch der UdSSR der Kapitalismus triumphiert, denke ich, daß die Zeichen anders gedeutet werden können: Da langfristig sozialistische Formen der gesellschaftlichen Umgestaltung wegen des Versagens der Marktgesetze unumgänglich werden, hat die sozialistische Theorie nun die vielleicht letzte - Chance, durch gründliche Verarbeitung der neoliberalen Theorie und ihrer teilweise zutreffenden Kritik die Voraussetzungen für ein funktionsfähiges Modell von "Dual-Wirtschaft" zu erarbeiten.

\section{Sozialistische Marktwirtschaft und "solare Revolution"}

In der "ökologischen" Perspektive gibt es eigentlich nur zwei wirkliche Revolutionen, die diesen großen Namen verdienen. Einmal die einige Jahrtausende zurückliegende "neolithische Revolution" (Gordon Child), in der die Menschen ihre symbiotische Existenz mit der Natur aufgegeben haben und von ihrer jagenden und sammelnden Lebensweise, bei der kleinste Gruppen extrem große Territorien benötigten, zur bewußten Nahrungsproduktion übergegangen sind. In dieser Zeit setzt das Wachstum der Erdbevölkerung ein. Es bedurfte mehrerer Jahrtausende, bis im 18. Jahrhundert die Grenze der ersten Milliarde erreicht war. Seitdem hat die demographische Entwicklung die Gestalt einer gefährlich wachsenden Kurve angenommen. Zwischen 1950 und 1994 hat sich die Bevölkerung des Planeten verdoppelt.

In der Soziologie von Marx spielt der Gegensatz von Stadt und Land eine große Rolle. Aber im Blick auf die Naturzerstörung gehören Stadt und Land zusammen. Gemeinsam haben die städtische und die ländliche Bevölkerung in Mesopothamien und in China, in Griechenland, Italien und Spanien, in Amerika und Afrika, die Wälder gerodet, die den Globus vorher bedeckt hatten. Dabei hat dieser unkoordinierte, aber gleichwohl gemeinsame Kampf um Ackerflächen, Rohmaterial und Energiequellen ökologische Katastrophen von gigantischem Ausmaß verursacht, die bis heute wirksam sind. 
Die menschliche Zivilisation verdankt der neolithischen Revolution unendlich viel. Der größte Teil unserer Werkzeuge, die Grundlagen der Keramik, der Metallurgie und der Weberei wurden damals erfunden. Seit diesem frühzeitlichen, unerhörten Innovationsschub ist in ökologischer Hinsicht die Richtung der Entwicklung gleichgeblieben. Die weiteren technologischen Revolutionen, die bereits im Mittelalter einsetzen, später die Entwicklung der Dampfkraft und die teilweise Ersetzung des Holzes als Energiequelle und Baumaterial durch Kohle und Eisen, haben die Stufenleiter der Ausbeutung der Erde nur immer weiter erhöht. In der Gegenwart mit ihren neuartigen Formen der Zusammenarbeit von Wissenschaft, Technik und Wirtschaft ist dieser Prozeß zur äußersten denkbaren Konsequenz vorangetrieben worden. Aber die Grundlinie der Ausbeutung der Erde hat sich nicht mehr verändert, sondern nur verschärft. Es wäre die Aufgabe einer sozialistischen Umgestaltung unserer Wirtschaft, die Ausplünderung der Erde und den räuberischen Kapitalismus zu überwinden und zu einer nachhaltigen Wirtschaftsweise überzugehen. Das Kernstück einer solchen fundamentalen Veränderung der Gesellschaft bestünde darin, die fossilen durch die solaren Energien zu ersetzen.

Aus den inzwischen allgemein bekanntgewordenen ökologischen Widerprüchen unserer Produktionsweise müssen harte Konsequenzen gezogen werden. Die nicht-erneuerbaren Ressourcen dürfen nur mit größter Sparsamkeit verwendet werden (Low-EntropyEconomy), und an Energien darf, wegen der katastrophalen Wirkung der angeblich billigen fossilen Energien, langfristig nur noch genutzt werden, was uns von der Sonne direkt oder indirekt zufließt. Eine solche "solare Strategie" und die volle und systematische Nutzung der "negativen Entropie" bietet auch praktisch die Chance, weltweit Wohlstand zu organisieren. Eine solche Zielsetzung ist weit davon entfernt, Armut und Unterentwicklung zu verharmlosen. Im Gegenteil: Es waren in den letzten vier Jahrzehnten die Muster eines verfehlten Modells der wirtschaftlichen Modernisierung, die den Abstand zwischen arm und reich im Weltmaßstab gefährlich vergrößert haben. Aber es muß neu definiert werden, worin "Wohlstand" und "Modernisierung" wirklich gesehen werden können. Eine solche Neubewertung muß von den Faktoren ausgehen, die für eine nachhaltige (sustainable), auf Dauer reproduktionsfähige menschliche Zivilisation entscheidend sind.

Es gibt viele wirtschaftliche Ziele, mit denen es gelingen könnte, ein Land zu "modernisieren", ohne dabei auf den Irrweg einer "Massenautomobilisierung" zu verfallen. Dazu gehören z.B.: gründliche Modernisierungen in Land- und Forstwirtschaft, ein umfassender sozialer Wohnungsbau und die Entwicklung moderner Solararchitektur, die Schaffung neuartiger Formen des Städtebaus, die dem Ziel der Verkehrsvermeidung untergeordnet sind, die Konstruktion moderner Formen des Massentransports von Personen und Gütern innerhalb und zwischen städtischen Zentren auf dem ökonomisch und ökologisch günstigen Schienenweg, der Ausbau vielfältiger Bereiche des Maschinenbaus, der Freigabe der Marktwirtschaft für alle Erfindungen auf dem Gebiet ökologisch unbedenklicher Industrien, vor allem aber die Umwelttechnologie selbst, der Aufbau moderner Formen der 
Telekommunikation mit dem Ziel, unnötigen Verkehr zu vermeiden, - um nur einige Beispiele zu geben.

Meines Wissens hat zuerst Georgescu-Roegen von einer "solaren Revolution" gesprochen. Sie würde einen vernünftigen "Stoffwechsel" zwischen Mensch und Natur wiederherstellen, wie es Marx und Engels einst vorgeschwebt hatte. Es würde eine grundlegende Umwälzung unserer Produktionsweise sein, wenn wir von nicht-erneuerbaren Rohstoffen und Energiequellen übergehen würden zu Rohstoffen und Energiequellen, die nachwachsen oder uns von außerhalb des Planeten zufließen. Ihr wesentliches Element wäre die Ersetzung der fossilen Energien durch Solarenergie. Hermann Scheer hat auch stichhaltige Argumente dafür entwickelt, daß sie in absehbarer Zeit möglich ist. ${ }^{4}$ Wenn eine solche solare Umwälzung unserer Produktionsweise auf neuer Basis etwas von der alten Symbiose von Mensch und Natur wiederherstellen würde, wie sie vor der neolithischen Revolution einmal bestanden hatte, so würde sie allerdings nun eine völlig andere Gestalt annehmen. Sie würde nicht bedeuten, in die Steinzeit zurückzukehren, wie manche Kritiker in unsachlicher Polemik behaupten. Im Gegenteil, Sonnenstrategie und Umweltpolitik stimulieren Beschäftigung und industrielle Innovation. Auf der Höhe moderner Wissenschaft und Technologie, würde sie mit allen Kenntnissen über die Möglichkeiten industrieller Produktion ausgestattet sein, die erforderlich ist, um für die Milliardenbevölkerung unseres Planeten die benötigten Gebrauchsgüter herzustellen. Die industrielle Gesellschaft läßt sich nicht überwinden, sie muß aber reorganisiert werden, indem ihr die Aufgabe gestellt wird, eine dauerhafte, nachhaltige Wirtschaftsweise zu entwickeln. Auch die nötigen öffentlichen Infrastrukturen des "Dualsystems" können nur aus ökonomischen Wertschöpfungsprozessen finanziert werden, was um so leichter gelingt, je stärker der Marktsektor der Wirtschaft eines Landes "floriert".

\subsection{Das breite Spektrum solarer Energie}

Das Projekt einer radikalen "solaren Wende" erscheint auf den ersten Blick utopisch. Aber wir sind bereits der Möglichkeit nahegekommen, ein so grundlegendes, neues Prinzip in der politischen Realität zu verwirklichen. Die solare Revolution ist inzwischen technisch und ökonomisch denkbar geworden. Der wichtigste Ausgangspunkt dafür, sich in der öffentlichen Diskussion überhaupt erst einmal verständlich zu machen, besteht darin, "solare" Energie richtig zu definieren. Zu ihr gehören im Sinne einer umfassenden Solarstrategie alle Energiequellen, die direkt oder indirekt auf die Sonneneinstrahlung zurückgehen. Neben den modernen Formen einer direkten Umwandlung der Sonnenenergie in elektrische Energie durch "Photovoltaik" steht die passive Erwärmung von Dächern und Fassaden durch die Sonnenwärme. Daneben treten alle Formen der Nutzung von

4 Hermann Scheer, Sonnenstrategie. Politik ohne Alternative, München: Piper, 1993, Übersetzungen in Englisch, Französisch, Spanisch. Hermann Scheer ist Mitglied des Bundestages, Präsident der Vereinigung "Eurosolar", die aktiv um die "solare Wende" in der Energie- und Wirtschaftspolitik kämpft, Mitherausgeber.des Yearbook of Renewable Energies. 
Wind und Wasser zur Energieerzeugung, weil diese Kräfte durch Erwärmung und Verdunstung entstehen, die die Sonne bewirkt hat. Schließlich haben uns die Pflanzen das faszinierende Phänomen der Photosynthese vorgemacht. Diese "Biomasse", die durch den Einfall von Sonnenlicht entsteht, kann als Rohstoff, aber auch in der Form von thermischer Energie genutzt werden, und zwar direkt durch Verbrennung oder indirekt als Biogas, das bei der Verrottung von Biomasse entsteht.

Eine Politik der bloßen Energieeinsparung allein kann die ökologischen Probleme nicht durchgreifend lösen; sie könnte, falls der Übergang zur Produktion neuer, regenerierbarer Energien nicht gelingen sollte, die Probleme nur für einige Zeit aufschieben. Falls die solare Revolution aber doch noch rechtzeitig verwirklicht werden könnte, käme der Energieeinsparung allerdings eine fundamentale Bedeutung zu. Denn wenn es, um nur einmal ein Zahlenbeispiel zu geben, nur möglich sein sollte, mit den solaren Energiequellen $50 \%$ der heute verwendeten Energien zu ersetzen, dann würden es $100 \%$ der benötigten Energien sein, falls es gelingen sollte, den Energieverbrauch gleichzeitig durch Sparmaßnahmen um 50\% zu senken. Die solare Zukunftsgesellschaft darf keine "Verschwendungsgesellschaft" sein, sondern muß sich in allen ihren Äußerungsformen darauf ausrichten, mit "niedriger Entropie" zu wirtschaften und keinen vermeidbaren Abfall zu produzieren.

\subsection{Photovoltaik}

Bei der Umwandlung von Sonnenstrahlung durch Photozellen in elektrischen Strom stoßen wir gleich auf einen Haupteinwand. Es wird die Ansicht vertreten und von den interessierten Machtstrukturen der Wirtschaft mit großem Aufwand verbreitet, daß die Solarenergie nur eine "zusätzliche", "additive" Energiequelle sein könne. Hermann Scheer weist detailliert nach, daß die nutzbare Menge der solaren Energie, die die Erde empfängt, bei weitem ausreicht, den gesamten Energiebedarf einer auf vernünftige Weise wohlhabenden Gesellschaft auf Dauer zu decken. Allerdings darf die Solarenergie dabei nicht nur auf die Photovoltaik setzen, sondern muß alle solaren Energiequellen miteinander kombinieren.

Die Photovoltaik hat die größten Chancen dort, wo der Energieeinsatz dezentral organisiert werden kann, etwa bei der Gebäudeheizung. Wo ein zentraler Energiebedarf nicht vermieden werden kann (Industrie, Elektrifizierung der Eisenbahn) müssen Zwischenschritte in Kauf genommen werden, die aber perspektivisch auf das langf ristige Hauptziel ausgerichtet sein müssen. Bei der vorläufigen weiteren Verwendung von Kohle müßte aber wenigstens darauf geachtet werden, den Wirkungsgrad zu erhöhen, indem systematisch durch Kraft-Wärme-Kopplung die Abwärme für Heizungszwecke genutzt wird. Kleinere Anlagen arbeiten heute im Blick auf diese Doppelnutzung rentabel und sollten den Großkraftwerken vorgezogen werden. Was den Flächenbedarf für Solarzellen angeht, so werden die Anforderungen oft übertrieben. Im Prinzip würde die Nutzung der bereits überbauten und versiegelten Flächen, namentlich der Dächer, durchaus reichen. Aller- 
dings muß dabei vorausgesetzt werden, daß systematisch der nicht benötigte dezentral erzeugte Strom in zentrale Energiespeicher abgegeben und in das Netz zurückgespeist wird. Da die Sonnenenergie nicht gleichmäßig anfällt, sondern starken Schwankungen im Tages- und im Jahresrhythmus ausgesetzt ist, muß eine Solarwirtschaft mit Speichermedien arbeiten, von denen die Wasserstoffproduktion durch solare Hydrolyse die größte Zukunftsaussicht hat.

\section{3. Passive Nutzung von Solarenergie}

Im Zeichen billiger Energien sind die Kenntnisse ganz vernachlässigt worden, wie man durch eine geeignete Architektur die einfallende Sonnenstrahlung nutzen und durch Wärmedämmung unnötige Energieverluste vermeiden kann oder sich vor der Erwärmung, wenn sie zu intensiv ist, schützt. Die Hochhäuser in Stahl-Glas-Bauweise haben einen Energiebedarf für Heizung, Kühlung und Infrastruktur, der geradezu abenteuerlich ist. Sie können im Blick auf die ökologisch relevanten Kriterien nicht mehr als "modern" gelten.

Im Gegensatz zu einem sogenannten "Funktionalismus", der die Funktion des Werkstoffes Glas, Wärme zu akkumulieren, nicht berücksichtigt hat, und diese energetisch wertvolle Funktion vielmehr durch aufwendige Heizungs- und Kühlungssysteme gerade wieder beseitigt, würde erst eine Solararchitektur "modern" und "funktional" sein. Sie würde sich die Kenntnisse wieder aneignen, die im Zeichen der angeblich "billigen" fossilen Energie in Vergessenheit geraten sind, wie in der Vergangenheit durch natürliche Bedingungen von Baumaterialien und Bauformen die Möglichkeiten der Erwärmung oder auch des Schutzes vor Sonnenstrahlung oder Auskühlung zum Teil geradezu genial genutzt worden sind. Daneben sind auf diesem Gebiet ganz neue Entwicklungen von Materialien zur Wärmedämmung, von neuen Techniken der Beleuchtung, der Heizung und vor allem der Konvertierung von Wärme und Kälte im Gange. Die stromfressenden elektrischen "Klimaanlagen" müßten, weil sie nach neueren Kriterien nicht mehr als "modern" gelten können und überflüssig sind, der Umweltverträglichkeitsprüfung zum Opfer fallen.

Alle Neubauvorhaben müßten unter den Aspekten des Energieverbrauchs geplant und geprüft werden. Es gibt bereits vielversprechende Ansätze einer neuartigen Solararchitektur" mit "Niedrig-Energie-Häusern", die sich bereits "rechnen" und die ihre ökologischen Mehrkosten in rationalen Zeiträumen amortisieren. Allerdings müßte die Solararchitektur mit dem "International Style" der immer noch vorherrschenden Architekturschulen brechen, der die größte Stadtzerstörung und Energieverschwendung der Geschichte zu verantworten hat. Es ist nicht zu verstehen, daß bei den heutigen gigantischen Bauvorhaben in Berlin die "Solararchitektur" nicht das beherrschende Tagesthema ist, obwohl ein Symposium über ein "Solares Regierungsviertel" viele Anstöße gebracht hatte. 


\subsection{Wind- und Wasserenergie}

Eine große Zukunftsbedeutung kommt schließlich der Stromerzeugung durch Windräder, Wasser-, Wellen- und Gezeitenkraftwerke zu, wobei in ökologischer Sicht die Vorteile von großen Staudämmen häufig durch extreme ökologische Nachteile aufgezehrt werden. Sie laufen auch insofern der solaren Strategie zuwider, als deren Ziel überwiegend darauf gerichtet ist, Energie dezentral zu erzeugen, um die mit der zentralen Energiewirtschaft verbundenen Großnetze nach Möglichkeit überflüssig zu machen. Eine Vielzahl von kleineren Wasserkraftwerken gilt ökologisch als vorzugswürdig. Vielversprechende Forschung ist bereits dabei, die Wirtschaftlichkeit kleiner und kleinster Anlagen entscheidend zu verbessern. Bei der Windenergie kann die moderne Technologie aus der Vergangenheit viele Anregungen ziehen, etwa aus den großen Leistungen beim Abpumpen von Wasser in den holländischen Deichanlagen. Auch bei der Windenergie ist die KostenNutzen-Bilanz bereits heute vielversprechend, vor allem, wenn sie von vornherein zusammen mit Rückeinspeisung in Speichermedien geplant wird.

\subsection{Biomasse}

Bei einem Übergang zur solaren Energieversorgung muß die gesamte verfügbare Biomasse, die durch pflanzliche Photosynthese entstanden ist, einschließlich der Biogase die bei ihrer Verrottung entstehen, systematisch und weltweit genutzt werden. Bei einem solchen umfassenden Konzept der "solaren Revolution" kommt einer modernen Forstwirtschaft eine herausragende Bedeutung zu. Die solare Strategie muß eine massive Wiederbegrünung der Erde postulieren. Weltweit ist in den nächsten Jahrzehnten eine Aufforstung von etwa 5 Mio km nötig (Scheer). Das würde bei den $130 \mathrm{Mio} \mathrm{km}_{2}^{2}$ der nicht mit Eis bedeckten Landfläche, von denen heute noch etwa 40 Mio km ${ }^{2}$ bewaldet sind, eine Vergrößerung der Wälder um mehr als $10 \%$ bedeuten. Man erschrickt vor diesen gewaltigen Zahlen. Aber die Flächen wären vorhanden: In Rußland wären verfügbar 17 Millionen $\mathrm{km}^{2}$, in China 9,6; in Brasilien 8,5; in Indien 3,3; in Argentinien 2,8; in Mexico 2 Millionen km . Einer solchen massiven Aufforstung käme eine vielfältige Bedeutung zu.

Zunächst würde eine solche weltweite Aufforstung während einer etwa vierzigjährigen Wachstumsperiode eine wichtige Funktion bei der Bindung von $\mathrm{CO}_{2}$ zukommen (Scheer). Neben den Weltmeeren mit ihrem Plankton sind es vor allem die Pflanzen der Erde, die bei ihrem Wachstum große Massen der von der Verbrennung von fossilen Energieträgern freigesetzten $\mathrm{CO}_{2}$-Mengen binden. Sie benötigen das Kohlendioxyd, um wachsen zu können und speichern es als Kohlenstoff, bis es durch Verbrennung wieder freigesetzt wird. Dabei entsteht ein Kohlendioxydkreislauf, der für die Umwelt an sich neutral und unschädlich ist. Bei einer massiven, globalen Aufforstung könnte der wachsende Wald große Mengen der gegenwärtig bei der Verbrennung fossiler Energieträger noch freigesetzten $\mathrm{CO}_{2}$-Mengen binden. Bei langlebigen Gegenständen, die aus Holz gefertigt werden, bleibt die $\mathrm{CO}_{2}$-Speicherung auch erhalten. Wenn aber die Solarstrategie anfängt zu 
greifen und die $\mathrm{CO}_{2}$-Belastung abgenommen hat, kann eine nachhaltige Forstwirtschaft das Holz auch thermisch verwerten.

Es kommen viele Nutzungen des nachwachsenden Rohstoffs Holz in Betracht. Er kann als Rohstoff der Holzchemie dienen, die gegenüber der Petrochemie große Vorteile zeigt. Während sich in den fossilen Rohstoffen massive Einlagerungen von Schwefel und anderen Schadstoffen finden, ist Holz ökologisch um einige Größenordnungen harmloser. Das gilt auch für die Verwendung von Holz bei der thermischen Verwertung. die bei geeigneten Verfahren (Mehrkammersysteme) hohe Wirkungsgrade zeigt.

Neben der aktiven Aufforstung müßte auch ein passiver Schutz der vorhandenen Wälder stehen. Nach Schätzungen der Weltbank fallen dem primären menschlichen Bedürfnis nach Energie für die Zubereitung von Nahrung täglich etwa $500 \mathrm{~km}^{2}$ Wald zum Opfer, das sind im Jahr etwa $180.000 \mathrm{~km}$ und damit jährlich etwa $0,5 \%$ der gesamten Waldfläche des Globus (Scheer). Um den Hunger nach Brennstoff zu befriedigen und diese Waldzerstörung zu beenden, sollten im Rahmen eines globalen Programms internationaler Hilfe, innerhalb kurzer Zeit die drei Milliarden Menschen, die ohne Elektrizität leben, mit solaren Kochgeräten ausgestattet werden. Sie haben sich bereits massenhaft bewährt und müßten primär in den betroffenen Ländern selbst hergestellt werden, wobei Handwerksbetriebe und kleine Industrien entstehen würden.

\section{Die mögliche Rolle von Verfassungs- und Verwaltungsrecht bei der Entwick- lung einer "sozialistischen Marktwirtschaft" und einer "sozialistischen Demo- kratie"}

Im System einer "sozialistischen" Marktwirtschaft müßte der öffentliche Sektor die Ziele festlegen, die für die Entwicklung des Gesamtsystems maßgebend sein sollen und für den Marktsektor klare, langfristig berechenbare und in sich stimmige Rahmenbedingungen schaffen. Hierbei kommt dem Verfassungsrecht und dem Verwaltungsrecht eine besondere Bedeutung zu.

Zum Schluß möchte ich wenigstens andeuten, in welcher Weise eine "sozialistische" Marktwirtschaft sich von einer "neoliberalen" und nur "sozialen" Marktwirtschaft zu ihrem Gunsten unterscheiden würde, wenn sie richtigen Gebrauch von der "Produktivkraft Wissenschaft" macht, die Instrumente des Verfassungs- und Verwaltungsrechts klug einsetzt und die Möglichkeiten einer "sozialistischen Demokratie" kreativ entwickelt. Eine kohärente Solarstrategie und das Ziel der Entwicklung einer nachhaltigen Wirtschaftsweise würden Dreh- und Angelpunkt einer solchen Modernisierung sein. Diese Umstellung in der Produktionsweise könnte und sollte ein gut durchdachtes Zusammenwirken mit modernisierten rechtlichen Strukturen und Verfahren wirksam unterstützen. 


\subsection{Die Rolle des Verfassungsrechts}

Aufgabe des Verfassungsrechts ist es, den demokratischen Prozeß dadurch zu strukturieren, daß bestimmte Errungenschaften, die das Ergebnis langer historisch-politischer Kämpfe waren, festgehalten und zur gemeinsamen Basis aller Kräfte des politischen Systems gemacht werden. So fixiert das Grundgesetz Deutschlands, um nur ein Beispiel zu geben, die republikanische Staatsform und verbietet von Verfassung wegen die Rückkehr zur Monarchie. Ferner verlangt sie von den politischen Parteien, den Verfassungskonsens einzuhalten (Art. 21 Abs. 2 GG). Auch die Bindung aller politischen Gewalt an bestimmte Grundrechte gibt dem politischen Prozeß bestimmte Rahmenbedingungen vor. $\mathrm{Da}$ es bei den Verfassungen nur eine begrenzte Zahl von organisatorischen Formen gibt, bestehen zwischen den Verfassungsstrukturen und den grundrechtlichen Standards einer bürgerlichen und einer sozialistischen Gesellschaft starke Elemente der Kontinuität (Joseph Schumpeter, Hermann Heller). Allerdings sind die organisatorischen Verfassungsstrukturen und die Grundrechte keine vom Himmel gefallenen Wahrheiten. Sie haben sich historisch entwickelt und unterliegen auch in Zukunft dem Wandel. Wenn man nicht an einem oberflächlichen Schlagabtausch zwischen unterschiedlichen Systemen interessiert ist, eröffnen sich fruchtbare Möglichkeiten, die überkommenen Formen des Verfassungsrechts unter sorgfältiger Anpassung an die jeweils spezifischen Bedingungen weiterzuentwickeln und mit ihrer Hilfe das politische und ökonomische System zu stabilisieren. Das wäre auch für die Zielsetzungen einer sozialistischen Marktwirtschaft unter den Rahmenbedingungen einer sozialistischen Demokratie möglich. Beim Übergang von einer Wirtschaftsweise der Raubbaus, die auf nicht-erneuerbaren Rohstoffen und Energiequellen beruht hat, zu einer wahrhaft nachhaltigen Wirtschaftsweise, können und müssen die neuen ökologischen Rahmenbedingungen und Vorgaben für das Wirtschaftssystem mit den Mitteln des Verfassungsrechts gesichert werden. Auch in der westlichen Verfassungsdiskussion spielt der grundrechtliche Schutz der ökologischen Lebensbedingungen der Gesellschaft eine wachsende Rolle.

\subsection{Die Rolle von Privatrecht und öffentlichem Recht}

Das Konzept einer "Dualwirtschaft" räumt gegenüber der Sozialismuskritik ein, daß ein vollständiger Übergang zu einer sozialistischen Gesellschaft nicht funktionieren kann. Einmal sind privatwirtschaftliche Unternehmen und Handlungsformen nach wie vor erforderlich, weil der Markt seine Aufgabe noch nicht erfüllt hat und im Blick auf die immensen Versorgungsprobleme der Milliardenbevölkerung der Erde auch nie erfüllt haben wird. Aufgaben von dieser Größenordnung müßte jede administrative Lösung "überfordern". Neben dem Marktsektor muß aber ein öffentlicher Sektor stehen, um das Marktversagen auszugleichen und dafür zu sorgen, daß die Bedingungen einer nachhaltigen Wirtschaftsweise erfüllt werden. Eine sozialistische Gesellschaft benötigt ein modernes Privatrecht, Gesellschaftsrecht, Versicherungsrecht, Patentrecht u.v.a.m. Zugleich braucht die öffentliche Hand aber auch ein ausgearbeitetes, differenziertes Verwaltungsrecht. Sie 
muß, ganz im Sinne Karl Renners, die Vorgaben, die im öffentlichen Interesse der Profitwirtschaft aufzuerlegen sind, in den Formen einer konsistenten Verwaltungsrechtsordnung entwickeln. Eine effektive Rechtsordnung ist auch erforderlich, um eine dezentrale Verwaltung aufzubauen. Nur dann, wenn die dezentralen Instanzen und die funktionell autonomen Entscheidungsträger "im Rahmen" des Rechts operieren, stärken die "Subsysteme" das Gesamtsystem und ruinieren es nicht.

\subsection{Die besondere Rolle der Gemeindedemokratie}

Bei einer ökologischen Umgestaltung der Gesellschaft kommt der Gemeindedemokratie eine besondere Bedeutung zu. Die ökologischen Rahmenbedingungen müssen zwar in der Form von Gesetzen von zentralen legislativen Instanzen entschieden werden. Aber die administrative Umsetzung hat einen stark lokalen Bezug. Nur Instanzen lokaler Demokratie sind geeignet, die Ziele des Gesamtsystems auf der örtlichen Ebene zu verstärken und selbständig umzusetzen. Bei großer räumlicher Entfernung und bei der Unübersichtlichkeit der Einzelkompetenzen können sich lokale administrative Instanzen relativ leicht der zentralen Kontrolle und den rechtlichen Standards entziehen. Eine wirkungsvolle Gemeindedemokratie ist die einzig bekannte Methode, überzentralisierte und ineffektive Kontrollmechanismen zu vermeiden und doch die lokalen Führungs- und Entscheidungsgremien an die gesetzlich definierten Ziele des Gesamtsystems zu binden.

Der Grund für diese Leistungsfähigkeit der Gemeindedemokratie besteht darin, daß die Gemeindebürger, die von den Entscheidungen der lokalen Verwaltungsinstanzen betroffen sind, aus Eigeninteresse stark motiviert sind, sich auf rechtliche Standards zu berufen, die zentrale Instanzen in ihrem Interesse gesetzt haben. Wenn sie nicht nur an die Kommunalaufsicht, sondern auch an unabhängige dezentrale Gerichtsinstanzen appellieren können, verstärken unabhängige Richter, die nur an die Gesetze gebunden sind, die geltenden Normen, auch außerhalb administrativer Kontrolle. Jede formale Organisation braucht Strukturen, die die Organisationsziele des Gesamtsystems, die sich auf dem Weg von oben nach unten normalerweise immer weiter abschwächen, wieder zu kräftigen. Im Zusammenwirken der Gemeindedemokratie mit Kommunalaufsicht und mit unabhängigen, dezentralen gerichtlichen Kontrollinstanzen kann eine solche Verstärkung der Organisationsziele gelingen. Die unmittelbare Kontrolle lokaler Verwaltungsinstanzen durch Bürger, die von ihren Entscheidungen betroffen sind, ist auch das einzig bekannte wirksame Mittel, um Geldverschwendung und Korruption zu bekämpfen.

\subsection{Rechtssätze und Rechtsgrundsätze}

Das sowjetische Sozialismusmodell ist unter anderem daran gescheitert, daß es die starken Elemente der Kontinuität in Wirtschaft und Recht zwischen bürgerlicher und sozialistischer Gesellschaft verkannt hatte. Aber nach seinem Zusammenbruch ist dieses falsch konstruierte System in das andere Extrem umgeschlagen. Es ist ein ganz übertriebenes 
Vertrauen in den Markt entstanden, das längst enttäuscht ist. Diese Enttäuschung hat im Bereich der gesellschaftlichen Beziehungen zu einer rücksichtslosen und zynischen "Ellenbogengesellschaft" geführt. Vor allem sind in einer Zeit, in der die alten Nationalstaaten längst erkannt haben, daß übernationale Zusammenarbeit erforderlich ist, rückwärtsgerichtete engstirnige nationalistische Regressionen ausgebrochen, die eigentlich für überwunden gehalten worden waren. Viele der Enttäuschten wünschen deshalb das untergegangene System zurück. Aber das ist keine Lösung. Es muß ein Weg nach vorn gefunden werden. Das ist nur möglich, wenn eine wirkliche Abbkehr von den Fehlkonstruktionen des untergegangenen Sozialismusmodells stattfindet. Auf der anderen Seite genügt es nicht, sich auf prinzipienlose Weise irgendwie "durchzuwursteln". Es ist von der größten Bedeutung, den Zynismus und die Regressionen zu überwinden und vor allem der jungen Generation eine Alternative zu der um sich greifenden Gleichgültigkeit aufzuzeigen.

Die Lösung dieser verwirrenden und komplizierten Fragen könnten in den Formen einer wahrhaft sozialistischen Marktwirtschaft und einer authentischen sozialistischen Demokratie gefunden werden, wenn sie zur radikalen Problematik der Ausbeutung vordringt, die im "Krieg gegen die Natur" liegt. Der Kapitalismus kann nicht in seiner ökonomischen Effizienz überboten werden. Der einzige Grund, auf das Konzept einer sozialistischen Transformation der Gesellschaft zurückzukommen, liegt darin, den ökologisch unhaltbar gewordenen Raubkapitalismus zu überwinden. Im Feuer der ökologischen Kritik kann die sozialistische Bewegung ihren verlorenen Enthusiasmus und ihre innere Siegesgewißheit wiedererlangen. Im Gegensatz zur bisherigen deutschen "sozialen Marktwirtschaft", die die Intervention des politischen Systems in die wirtschaftlichen Abläufe so gut wie möglich zu verstecken sucht, würde eine "sozialistische" Marktwirtschaft die Funktion des politischen Systems offen und offensiv vertreten, um Rahmenbedingungen zu schaffen, die eine dauerhafte, nachhaltige Wirtschaftsordnung garantieren und die der Markt mit seinem Zwang zum permanenten Wirtschaftswachstum selbst nicht erfüllt.

Es ist eine positive Ernüchterung, wenn ein Sozialismus-Modell die Dualwirtschaft bejaht und den Marktsektor für sinnvoll und erforderlich erklärt. Damit kann dem Utopismus entgegengewirkt werden, der sich den Sozialismus in der Art eines Wunderglaubens in quasi-religiösen Farben ausgemalt hatte. Das Konzept einer sozialistischen Marktwirtschaft ist gegenüber den übertriebenen Ideen ein pragmatisches, wirklich erreichbares Ziel. Das Konzept einer sozialistischen Marktwirtschaft ist auch dort pragmatisch, wo sie mit der "solaren Wende" ein faszinierendes, über den Tageshorizont hinausgehendes Ziel aufstellt. Denn trotz ihres weitgespannten Ziels hat auch die "solare Revolution" einen pragmatischen Zug. Sie ist an jedem kleinen praktischen Einzelschritt interessiert, überflüssigen Abfall zu vermeiden, Reststoff wiederzugewinnen und die Verschwendung von Energie und Belastungen der Umwelt zu vermeiden. Aber auf der anderen Seite setzt die "solare Revolution" ein Ziel, das die ganze Vorstellungskraft erfüllt, Energien freisetzt und den Einsatz der Besten lohnt. Es gibt keine höhere Aufgabe, als den Krieg gegen die 
Natur zu beenden und einen vernünftigen Stoffwechsel des Menschen mit der Natur wiederherzustellen. So dient die "solare Revolution" beiden Zielen: Sie ernüchtert und begeistert. Damit bietet sie vor allem auch der desillusionierten jungen Generation eine Perspektive, die sich lohnt: eine Welt zu schaffen, die Bestand hat.

Solche weitreichenden Ziele können nur durch die kollektive Anstrengung zahlreicher Einzelner, Institutionen und Forschungseinrichtungen präzise entwickelt und umgesetzt werden. Es sind aber bereits viele am Werk. Es geht heute darum, die vielen einzelnen Elemene in ein umfassendes, kohärentes System zu integrieren und in der internationalen Zusammenarbeit den bereits erreichten Wissensstand besser zu nutzen. Es entstehen einer Volkswirtschaft unvertretbare Kosten, wenn sie nicht methodisch versucht, aus den Fehlern anderer zu lernen und Entwicklungen zu vermeiden, die in den als Vorbild betrachteten Ländern von den weitsichtigen Gruppen dort bereits als Fehlentwicklung eingeschätzt werden. Hermann Heller hat in seiner Staatslehre (1934) gezeigt, daß ein gesellschaftliches System und eine Rechtsordnung sich auf Dauer nur dann behaupten können, wenn sie in tief verwurzelten "Rechtsgrundsätzen" verankert sind und die einzelnen sich innerlich verpflichtet fühlen, das, was sie theoretisch erkennen, auch praktisch zu leben. Das faszinierende Ziel der solaren Revolution und des Übergangs zu einer nachhaltigen Wirtschaftsweise, die die jahrtausendealte Raubbauwirtschaft beendet, die mit der neolithischen Revolution begann, kann die inneren Auflösungstendenzen unserer Gesellschaften überwinden, der Demokratiebewegung eine deutliche Richtung zeigen und die erforderliche gesellschaftliche Integration bewirken. Wenn eine "sozialistische Marktwirtschaft" sich auf diesen Weg begibt, kann sie sich gegenüber dem neoliberalen Kapitalismus, aber auch gegenüber der nur "sozialen" Marktwirtschaft als überlegen zeigen und sogar weltweit die Perspektive einer "Konvergenztheorie" entwickeln, um die erstorbenen sozialistischen Ideen in den hochindustrialisierten Staaten wiederzubeleben, die für den Umbau in eine nachhaltige, dauerhafte Gesellschaft, im Rahmen einer solare Strategie gewonnen werden können, zu der es, wenn sich die Menschheit nicht aufgeben will, keine Alternative gibt (Hermann Scheer). 
restored, it is suggested that the people of Australia will suffer. They will lose the precious value of decision-makers who are independent of government. That independence has, until now, been a mainstay of liberty in Australia.

\section{Social or Socialistic Possibilities of Market Economy - Economic Development Through Constitutional and Administrative Law}

\section{By Christoph Müller}

After the internal collapse of the former USSR, it seemed to many that capitalism would then triumph worldwide. However, in no country in the world does there exist a pure market economy. Rather, a "mixed economy" is in existence almost everywhere. In the economy, a private sector and a public sector are to be found, with the latter regulating the structural conditions of the system through infrastructure policy, intervening in various ways in the economy, and participating directly in economic life in the form of public utilities. The systems of today can only be differentiated by considering the respective size of the two sectors (private and public) and what goals the public sector hopes to achieve. In a system of "socialistic" market economy, the public sector must assert those aims of development conducive to public wellbeing, and create clear and consistent perspectives and conditions for the private sector. In this paper, some practical and realizable examples will try to demonstrate how a "socialistic" market economy could be advantageously different from a "neoliberal" or only "social" market economy if it makes correct use of the "productive force of science", intelligent use of the instruments of constitutional and administrative law, and creative use of the possibilities of a socialistic democracy.

\section{Review of Regulations in the People's Republic of China}

\section{By Anke Frankenberger}

Administrative regulations are a feature of modern societies that is growing in number and complexity. In China the most obvious distinction in administrative regulations is between fagui and guizhang. Regulations in the PRC are characterized by multiple conflicts among them, and between them and laws and the constitution.

Since 1982 China has built up its legal system and in the last five years has enacted several laws and regulations concerning the review of administrative actions. There are 